\title{
Finite element heterogeneous multiscale method for Elastic Waves in Heterogeneous Media
}

\author{
A. Abdulle, M. J. Grote and O. Jecker
}

Departement Mathematik und Informatik

Preprint No. 2018-05

February 2018

Fachbereich Mathematik

Universität Basel

CH-4051 Basel

www.math.unibas.ch 


\title{
Finite element heterogeneous multiscale method for Elastic Waves in Heterogeneous Media
}

\author{
Assyr Abdulle ${ }^{\mathrm{a}}$, Marcus J. Grote ${ }^{\mathrm{b}}$, Orane Jecker ${ }^{\mathrm{a}}$ \\ ${ }^{a}$ École Polytechnique Fédérale de Lausanne, Switzerland \\ ${ }^{b}$ Universität Basel, Switzerland
}

\begin{abstract}
A finite element heterogeneous multiscale method (FE-HMM) is proposed for the simulation of time-dependent elastic waves in a rapidly varying heterogeneous elastic medium. It is based on a standard finite element discretization of an effective wave equation at the macro scale, whose a priori unknown effective material coefficients are computed on sampling domains at the micro scale within each macro finite element. Hence the computational effort becomes independent of the highly heterogeneous elastic medium at the smallest scale. Optimal error estimates and convergence rates in the energy and the $L^{2}$ norm are derived, which are explicit in the macro and micro discretization errors. Numerical experiments verify the sharpness of the error bounds and illustrate the versatility of the method for non-periodic, layered or stochastic media.
\end{abstract}

Keywords: multiscale methods, heterogeneous media, wave equation, linear elasticity, numerical homogenization, upscaling

\section{Introduction}

The efficient numerical simulation of time-dependent elastic wave phenomena is of fundamental importance in a variety of scientific and engineering applications. Finite element methods (FEM) are becoming increasingly popular because they easily accommodate localized small scale geometric features such as cracks, fractures, pinch-outs, or material interfaces. Thus when combined with local time-stepping strategies [20, 27], high-order FE methods are probably the method of choice for elastic wave propagation [36].

Yet when material heterogeneities not only occur at a scale much smaller than the wave length but also throughout the computational domain, classical finite element (or finite difference) methods become inefficient. As standard FE methods require grid resolution down to the finest scales in the medium, they indeed lead to prohibitively large problem sizes, even though the wave length itself might occur at a more moderate macroscopic scale. Not only for the simulation of seismic waves at the planetary scale but also for the prediction of the complex time-dependent response of engineered composite materials or structures, the need for multiscale strategies becomes all too obvious.

Multiscale methods generally fall into either of two classes: with or without explicit scale separation. Multiscale methods that forgo any underlying assumption of scale separation 
either replace the medium by an upscaled effective medium or devise a generalized multiscale FE basis constructed to capture the fine-scale information. This preprocessing step typically requires the solution of numerous local problems whose computational cost scales as the original problem for decreasing $\varepsilon$.

In the absence of scale separation, Capdeville et al. [14, 15], for instance, extended classical two-scale homogenization to the non-periodic case by artificially introducing a small scale parameter, $\varepsilon_{0}$, as the ratio between the desired (user-defined) smallest length scale and the smallest physical wave length. After a standard two-scale asymptotic expansion in $\varepsilon_{0}$, the resulting local cell problems are either solved by FE [14] or by FFT [15]. Finally, a low-pass filter is applied to the resulting stresses and strains to build a smoothly varying effective elastic tensor, which can then be used within any standard FE method.

Alternatively, multiscale FE or FD methods determine during pre-processing new multiscale FE or FD basis functions by numerically solving appropriate local problems. In particular, we mention the operator upscaling method proposed in [38], which is based on a two stage procedure. The computational domain is first decomposed into a macro mesh and subgrid problems resolving the smallest scale are computed on each macro element. Subgrid solutions and coarse test functions are then used to obtain the solution of the wave equation. Another related method is based on generalized FE bases [24, 25]. As in [38], a macro mesh is first defined and multiscale basis functions are computed within each coarse block to form a basis for computing a solution of the wave equation. Without the assumption of scale separation, those fine scale problems need to be resolved inside each macro element; hence, the computational cost of such operator-based upscaling methods typically scales like a fully resolved FE (or FD) method. Moreover, non-standard (generalized) FE bases not only lead to denser stiffness matrices but also to mass matrices, which cannot be lumped into diagonal approximations anymore $[24,38]$. This difficulty is somewhat mitigated by the use of discontinuous Galerkin methods which yield a block-diagonal mass-matrix [25]. By pre-computing associated harmonic coordinate transformations, one can also avoid the assumption of scale separation; nevertheless multiple fine scale problems then need to be solved over the entire computational domain [35]. For further references on multiscale methods for wave propagation, we refer to the recent review article [8].

In contrast, the finite element heterogeneous mutiscale method (FE-HMM) assumes that the heterogeneities occur at a micro scale $\varepsilon>0$ smaller than the dominating wave length. Still, it neither requires the small-scale dependence to be periodic nor does it make any simplifying assumptions about the macro scale behavior of the elastic medium, which may also contain isolated small scale features. The heterogeneous multiscale method (HMM) was first introduced for elliptic multiscale problems $[21,4,5]$ and computes an effective macro equation from micro computations in local sampling domains of size $\varepsilon$; hence, the computational cost remains independent of the micro scale.

For stationary multiscale problems from elasticity, the FE-HMM was proposed and analyzed in [1] while numerical examples were given in [9]. The FE-HMM for the acoustic wave equation was first introduced in [6] for moderate fixed times $T>0$ and later extended in [7] to long time. Indeed at very long times, $T^{\varepsilon}=\varepsilon^{-2} T$, the true solution begins to deviate from 
the homogenized equation, as it develops a dispersive behavior due to the subtle interplay between small and intermediate scales. To capture this dispersive effect, the homogenized model requires additional terms which lead to a family of effective Boussinesq-type equations $[7,10]$. An alternative finite difference based multiscale method for the long time wave propagation in heterogeneous media was proposed in $[22,23]$, which uses dynamical micro problems. In one dimension and for periodic media, the method given in [12] captures the effective flux of the long time multiscale wave problem.

Here, we propose and analyze the first FE-HMM for time-dependent elastic waves. From a rigorous and fully discrete a priori error analysis, we infer optimal settings for the macro/microscopic mesh parameters. Moreover, the complexity of the FE-HMM method scales only with the number of macro degrees of freedom, that is independently of $\varepsilon$. Various numerical examples in layered, stochastic or fully heterogeneous media demonstrate both the accuracy and efficiency of the FE-HMM for numerical wave propagation of elastic waves in arbitrary heterogeneous media.

Our paper is structured as follows. In Section 2, we introduce the elastic wave equation and recall some known analytical results from classical homogenization theory. Next in Section 3, we present the FE-HMM for the elastic wave equation and derive in Section 4 optimal error estimates with respect to the $L^{2}$ and the energy norm. Finally, we present some numerical experiments in Section 5 to corroborate the theory and to illustrate the versatility of the FE-HMM approach in the presence of non-periodic, anisotropic or stochastic media.

Notations. Here $C>0$ denotes a generic constant independent of $\varepsilon$ and whose value can change at any occurrence. For $r=\left(r_{1}, \ldots, r_{d}\right) \in \mathbb{R}^{d}$ we let $|r|=r_{1}+\ldots+r_{d}$ and $D^{r}=$ $\partial_{1}^{r_{1}} \ldots \partial_{d}^{r_{d}}$. We consider the usual Sobolev space $H^{1}(\Omega)=\left\{u \in L^{2}(\Omega)\left|D^{r} u \in L^{2}(\Omega),\right| r \mid \leq 1\right\}$ equipped with the norm $\|u\|_{\mathrm{H}^{1}(\Omega)}=\left(\sum_{|r| \leq 1}\left\|D^{r} u\right\|_{\mathrm{L}^{2}(\Omega)}^{2}\right)^{1 / 2}$ and denote by $H_{0}^{1}(\Omega)$ the closure of $\mathcal{C}_{0}^{\infty}(\Omega)$ w.r.t. the $H^{1}$ norm. We also denote by $Y=(0,1)^{d}$ the unit cube in $\mathbb{R}^{d}$ and let $W_{\text {per }}^{1}(Y)=\left\{u \in H_{\text {per }}^{1}(Y) \mid \int_{Y} u \mathrm{~d} y=0\right\}$, where $H_{\text {per }}^{1}(Y)$ is defined as the closure of $\mathcal{C}_{\text {per }}^{\infty}(Y)$ w.r.t. the $H^{1}$ norm.

\section{The elastic wave equation}

We consider a linear highly heterogeneous elastic medium contained in a bounded domain $\Omega \subset \mathbb{R}^{d}, d=1,2,3$ with Lipschitz continuous boundary $\Gamma$. Inside $\Omega$, we assume that the heterogeneities occur at a micro scale $\varepsilon>0$ smaller than the dominating wave length but make no further simplifying assumptions, such as periodicity, about the medium. Hence, we seek the displacement $u^{\varepsilon}:[0, T] \rightarrow H_{0}^{1}(\Omega)^{d}$ governed by the elastic wave equation

$$
\begin{aligned}
\partial_{t t} u^{\varepsilon}(t)-\operatorname{div}\left(a^{\varepsilon}(x): e\left(u^{\varepsilon}(t)\right)\right) & =f(t), & & \text { in } \Omega \times(0, T], \\
u^{\varepsilon}(t) & =0, & & \text { on } \Gamma \times[0, T],
\end{aligned}
$$

with the initial conditions at time $t=0$,

$$
u^{\varepsilon}(0)=g_{1}, \quad \partial_{t} u^{\varepsilon}(0)=g_{2}, \quad \text { in } \Omega .
$$


Here, $u^{\varepsilon}(t)$ associates to every time $t$ the spatially varying $d$-dimensional vector-valued displacement field of the elastic medium, $\left[u_{1}^{\varepsilon}(t, x), \ldots, u_{d}^{\varepsilon}(t, x)\right]^{\top}$, whereas $f=\left(f_{1}(t, x), \ldots, f_{d}(t, x)\right)$ and $g_{i}=\left(g_{i_{1}}(x), \ldots, g_{i_{d}}(x)\right)$ for $i=1,2$. For conciseness we will sometimes omit the explicit dependency on time and merely use $u^{\varepsilon}$ to denote $u^{\varepsilon}(t)$. In (1), the linearized $d \times d$ strain tensor $e$ is defined as

$$
e\left(u^{\varepsilon}\right)=\left(e_{i j}\left(u^{\varepsilon}\right)\right)_{1 \leq i, j \leq d}, \quad e_{i j}\left(u^{\varepsilon}\right)=\frac{1}{2}\left(\frac{\partial u_{i}^{\varepsilon}}{\partial x_{j}}+\frac{\partial u_{j}^{\varepsilon}}{\partial x_{i}}\right), \quad i, j=1, \ldots, d .
$$

The final time $T>0$ is fixed independently of $\varepsilon$. Otherwise the true solution $u^{\varepsilon}$ displays at very long times, $T^{\varepsilon}=\varepsilon^{-2} T$, a dispersive behavior no longer described by classical homogenization theory. Additional terms are then needed to capture those dispersive effects, leading to a family of effective Boussinesq-type equations $[7,10]$.

The weak formulation of (1) reads: find $u^{\varepsilon}:[0, T] \rightarrow H_{0}^{1}(\Omega)^{d}$ such that

$$
\left\langle\partial_{t t} u^{\varepsilon}(t), w\right\rangle+B^{\varepsilon}\left(u^{\varepsilon}(t), w\right)=F(w, t), \quad \forall w \in H_{0}^{1}(\Omega)^{d},
$$

with the initial conditions $(2)$. Here $\langle\cdot, \cdot\rangle$ denotes the dual pairing between $H^{-1}$ and $H^{1}$ (if $\partial u^{\varepsilon}(t)$ is in $L^{2}(\Omega)$, we use the standard $L^{2}$ inner product) whereas the bilinear form $B^{\varepsilon}: H^{1}(\Omega)^{d} \times H^{1}(\Omega)^{d} \rightarrow \mathbb{R}$ is given by

$$
B^{\varepsilon}(v, w)=\int_{\Omega} a^{\varepsilon}(x) e(v): e(w) \mathrm{d} x,
$$

and the right hand side $F: H^{1}(\Omega)^{d} \rightarrow \mathbb{R}$ is given by

$$
F(w, t)=\int_{\Omega} f(t) w \mathrm{~d} x
$$

For any $d \times d$ square matrix $M$, we denote its Frobenius norm by $\|M\|_{F}=(M: M)^{1 / 2}=$ $\left(\sum_{i, j=1}^{d} M_{i j}^{2}\right)^{1 / 2}$. Then, we assume that the fourth-order heterogeneous tensor $a^{\varepsilon}, a_{i j k l}^{\varepsilon}(x) \in$ $L^{\infty}(\Omega)$, for $i, j, k, l=1, \ldots, d$ satisfies

$$
\begin{aligned}
a_{i j k l}^{\varepsilon} & =a_{j i k l}^{\varepsilon}=a_{k l i j}^{\varepsilon}, \\
\alpha\|M\|_{F}^{2} & \leq a^{\varepsilon} M: M, \text { for any symmetric matrix } M, \\
\left\|a^{\varepsilon} M\right\|_{F} & \leq \beta\|M\|_{F}, \text { for any symmetric matrix } M,
\end{aligned}
$$

where $0<\alpha \leq \beta<\infty$. Under the above assumptions, the weak formulation (3) is well-posed and thus admits a family of unique solutions $\left\{u^{\varepsilon}\right\}_{\varepsilon}$, indexed by the superscript $\varepsilon$. Indeed, by combining the first Korn inequality,

$$
\|v\|_{\mathrm{H}^{1}(\Omega)} \leq C\left(\int_{\Omega}|e(v)|^{2} \mathrm{~d} x\right)^{1 / 2},
$$

where the $H^{1}(\Omega)^{d}$ norm is defined as

$$
\|v\|_{\mathrm{H}^{1}(\Omega)}=\left(\sum_{i, j=1}^{d} \int_{\Omega}\left(\frac{\partial v_{i}}{\partial x_{j}}\right)^{2} \mathrm{~d} x+\sum_{i=1}^{d} \int_{\Omega} v_{i}^{2} \mathrm{~d} x\right)^{1 / 2}
$$


with the coercivity of the the tensor $a^{\varepsilon}$, we immediately conclude that the bilinear form $B^{\varepsilon}$ is coercive, i.e.

$$
B^{\varepsilon}(v, v)=\int_{\Omega} a^{\varepsilon}(x) e(v): e(v) \mathrm{d} x \geq C\left(\int_{\Omega}|e(v)|^{2} \mathrm{~d} x\right) \geq C\|v\|_{\mathrm{H}^{1}(\Omega)}^{2}, \quad \forall v \in H_{0}^{1}(\Omega)^{d} .
$$

The existence and uniqueness of a (weak) solution, given sufficient regularity of the data,

$$
f \in L^{2}\left(0, T ; L^{2}(\Omega)^{d}\right), \quad g_{1} \in H_{0}^{1}(\Omega)^{d}, \quad \text { and } \quad g_{2} \in L^{2}(\Omega)^{d},
$$

follows then from the Lax-Milgram's Lemma and

$$
u^{\varepsilon} \in L^{2}\left(0, T ; H_{0}^{1}(\Omega)^{d}\right) \quad \text { and } \quad \partial_{t} u^{\varepsilon} \in L^{2}\left(0, T ; L^{2}(\Omega)^{d}\right) .
$$

In fact, $u^{\varepsilon}$ is more regular (see [31]) because $u^{\varepsilon} \in L^{\infty}\left(0, T ; H_{0}^{1}(\Omega)^{d}\right)$ with time derivative $\partial_{t} u^{\varepsilon} \in L^{\infty}\left(0, T ; L^{2}(\Omega)^{d}\right)$. Therefore, we have

$$
u^{\varepsilon} \in \mathcal{C}\left([0, T] ; H_{0}^{1}(\Omega)^{d}\right) \quad \text { and } \quad \partial_{t} u^{\varepsilon} \in \mathcal{C}\left([0, T] ; L^{2}(\Omega)^{d}\right) .
$$

\section{Classical homogenization theory}

The numerical solution of (1) with standard FEM requires the mesh size to be smaller than the finest scales in the medium, which is prohibitively expensive when $\varepsilon$ is small. Still, the effective dynamics at the macro scale can be described using homogenization theory $[34,19]$. By using the theory of $H$-convergence $[32,11]$, one can show that the effective behavior of the heterogeneous solution $u^{\varepsilon}$ is well-described by the solution of the homogenized wave equation for short times $T>0[13,19]$. Indeed, $u^{\varepsilon}$ converges (up to a subsequence) weakly in $H^{1}$ to the solution, $u^{0}$, of

$$
\left\langle\partial_{t t} u^{0}(t), w\right\rangle+B^{0}\left(u^{0}(t), w\right)=F(w), \quad \forall w \in H_{0}^{1}(\Omega)^{d},
$$

with the initial conditions (2). Here the bilinear form $B^{0}: H^{1}(\Omega)^{d} \times H^{1}(\Omega)^{d} \rightarrow \mathbb{R}$ is given by

$$
B^{0}(v, w)=\int_{\Omega} a^{0}(x) e(v): e(w) \mathrm{d} x
$$

where the homogenized tensor $a^{0}$ satisfies properties (4)-(6) for some constants $0<\alpha_{0} \leq$ $\beta_{0}<\infty$. Under additional assumptions on the small scale behavior of the tensor, such as periodicity

(H1) $a^{\varepsilon}(x)=a(x / \varepsilon)=a(y)$ is $Y$-periodic in $y$, where $Y=(0,1)^{d}$,

the whole sequence $u^{\varepsilon}$ converges weakly to $u^{0}$ and explicit equations are available to compute $a^{0}$ :

$$
a_{i j k l}^{0}=\frac{1}{|Y|} \int_{Y} a_{i j k l}(y)+\sum_{h, m=1}^{d} a_{i j h m}(y) \frac{\partial \chi_{h}^{k l}(y)}{\partial y_{m}} \mathrm{~d} y,
$$


where the functions $\chi_{h}^{k l} \in W_{p e r}(Y)$ are solutions of the micro problems

$$
-\frac{\partial}{\partial y_{j}}\left(a_{i j h m} \frac{\partial \chi_{h}^{k l}}{\partial y_{m}}\right)=\frac{\partial a_{i j k l}}{\partial y_{j}}, \quad \text { in } Y, \quad \text { for } i=1, \ldots, d,
$$

with periodic boundary conditions. Here, the space $W_{\text {per }}(Y)$ is defined as

$$
W_{\text {per }}(Y)=\left\{v \in H_{p e r}^{1}(Y)^{d} \mid \int_{Y} v_{i} \mathrm{~d} y=0, i=1, \ldots, d\right\} .
$$

Remark. Problem (1) can be easily adapted to inhomogeneous Dirichlet and Neumann boundary conditions. Then lifting of the Dirichlet data is required, which leads to additional terms in (3) and (8).

\section{FE-HMM for elastic waves}

The FE-HMM approach incorporates micro to macro scale interactions by solving inside each element at the coarse level a few micro scale problems inside sampling domains centered about quadrature nodes. By averaging the solutions of those micro problems, the overall effects are then translated from micro to macro scale. Thus we consider a family of regular coarse partitions $\left\{\mathcal{T}_{H}\right\}$ over $\Omega$ with mesh size $H \gg \varepsilon$ and introduce the corresponding standard continuous piecewise polynomial (macro) FE space of degree $p \geq 1$ :

$$
V^{p}\left(\Omega, \mathcal{T}_{H}\right)=\left\{v^{H} \in H_{0}^{1}(\Omega)^{d}\left|v^{H}\right|_{K} \in \mathcal{R}^{p}(K)^{d}, \forall K \in \mathcal{T}_{H}\right\},
$$

where $\mathcal{R}^{p}(K)$ is the space $\mathcal{P}^{p}(K)$ of polynomials on $K$ of degree at most $p$ if $K$ is a triangle (or tetrahedron), or the space $\mathcal{Q}^{p}(K)$ of polynomials on $K$ of degree at most $p$ in each variables if $K$ is a rectangle (or hexahedron).

For computation, the integrals involved in the finite-dimensional Galerkin counterpart of (3) must be approximated numerically. In doing so, we shall use two different quadrature formulas $(\mathrm{QF})$, the first to approximate the bilinear form and the second to approximate the $L^{2}$ inner product. Each QF needs to satisfy separate accuracy and stability requirements to avoid any subsequent loss in accuracy and thus achieve optimal convergence rates.

First, let $\hat{K}$ be a reference element and $\left\{\hat{\omega}_{j}, \hat{x}_{j}\right\}$ be any QF on $\hat{K}$. We shall make some or all of the following assumptions; for $j=1, \ldots, J$, with $J \geq 1$, let $\hat{\omega}_{j}>0$ and

(Q1) there exists $\lambda>0: \sum_{j=1}^{J} \hat{\omega}_{j}\left|\nabla \hat{p}\left(\hat{x}_{j}\right)\right|^{2} \geq \lambda\|\nabla \hat{p}\|_{\mathrm{L}^{2}(\hat{K})}^{2}$, for all $\hat{p} \in \mathcal{R}^{p}(\hat{K})^{d}$,

(Q2) $\int_{\hat{K}} \hat{p}(\hat{x}) \mathrm{d} \hat{x}=\sum_{j=1}^{J} \hat{\omega}_{j} \hat{p}\left(\hat{x}_{j}\right)$, for all $\hat{p} \in \mathcal{R}^{\sigma}(\hat{K})^{d}$,

where $\sigma=\max (2 p-2, p)$ if $\mathcal{R}^{\sigma}=\mathcal{P}^{\sigma}$, or $\sigma=\max (2 p-1, p+1)$ if $\mathcal{R}^{\sigma}=\mathcal{Q}^{\sigma}$.

(Q3) $\sum_{j=1}^{J} \hat{\omega}_{j}\left|\hat{p}\left(\hat{x}_{j}\right)\right|^{2} \geq \lambda\|\hat{p}\|_{\mathrm{L}^{2}(\hat{K})}^{2}$, for all $\hat{p} \in \mathcal{R}^{p}(\hat{K})^{d}$. 
Inside each element $K$, we now consider two different QF, $\left\{\omega_{j, K}, x_{j, K}\right\}$ and $\left\{\tilde{\omega}_{l, K}, \tilde{x}_{l, K}\right\}$ with $1 \leq j, l \leq L$, to evaluate the bilinear form $B_{H}$ and the discrete inner product $(\cdot, \cdot)_{H}$, respectively. We assume that both QF satisfy assumptions (Q1) and (Q2) above, while the second $\mathrm{QF}$, used to evaluate $(\cdot, \cdot)_{H}$, must also satisfy assumption (Q3).

Around each quadrature node $x_{j, K}$ involved in the numerical approximation of $B_{H}$, we consider a sampling domain $K_{\delta_{j}}$ of size $\delta \ll H$ and a family of fine partitions $\left\{\mathcal{T}_{h}\right\}$ over $K_{\delta_{j}}$ of mesh size $h \leq \varepsilon$. There we also introduce the corresponding micro FE space of continuous piecewise polynomials of degree $q \geq 1$ :

$$
V^{q}\left(K_{\delta_{j}}, \mathcal{T}_{h}\right)=\left\{v^{h} \in W\left(K_{\delta_{j}}\right)\left|v^{h}\right|_{K} \in \mathcal{R}^{q}(K)^{d}, \forall K \in \mathcal{T}_{h}\right\} .
$$

The choice of the subspace $W\left(K_{\delta_{j}}\right)$ of $H^{1}\left(K_{\delta_{j}}\right)^{d}$ determines the coupling between the micro and macro solvers by incorporating the appropriate boundary conditions into the micro problems (14) below, namely

$$
\begin{array}{ll}
W\left(K_{\delta_{j}}\right)=H_{\mathrm{per}}^{1}\left(K_{\delta_{j}}\right)^{d}, & \text { for periodic coupling, } \\
W\left(K_{\delta_{j}}\right)=H_{0}^{1}\left(K_{\delta_{j}}\right)^{d}, & \text { for Dirichlet coupling. }
\end{array}
$$

Given the above definitions of the macro and micro FE spaces, the overall FE-HMM reads:

find $u^{H} \in V_{0}^{p}\left(\Omega, \mathcal{T}_{H}\right)$ such that

$$
\left(\partial_{t t} u^{H}, w^{H}\right)_{\mathrm{H}}+B_{H}\left(u^{H}, w^{H}\right)=F\left(w^{H}\right), \quad \forall w^{H} \in V_{0}^{p}\left(\Omega, \mathcal{T}_{H}\right),
$$

with initial conditions given by nodal interpolations of $g_{1}$ and $g_{2}$ given in (2). We define $(\cdot, \cdot)_{\mathrm{H}}$ by

$$
\begin{aligned}
\left(\partial_{t t} v^{H}, w^{H}\right)_{\mathrm{H}} & =\sum_{K \in \mathcal{T}_{H}} \sum_{l=1}^{L} \tilde{\omega}_{l, K} \partial_{t t} v^{H}\left(\tilde{x}_{l, K}\right) w^{H}\left(\tilde{x}_{l, K}\right), \\
F\left(w^{H}\right) & =\int_{\Omega} f w^{H} \mathrm{~d} x .
\end{aligned}
$$

The bilinear form $B_{H}: V_{0}^{p}\left(\Omega, \mathcal{T}_{H}\right) \times V_{0}^{p}\left(\Omega, \mathcal{T}_{H}\right) \rightarrow \mathbb{R}$, in (12), is given by

$$
B_{H}\left(u^{H}, w^{H}\right)=\sum_{K \in \mathcal{T}_{H}} \sum_{j=1}^{J} \frac{\omega_{j, K}}{\left|K_{\delta_{j}}\right|} \int_{K_{\delta_{j}}} a^{\varepsilon}(x) e\left(u_{j}^{h}\right): e\left(w_{j}^{h}\right) \mathrm{d} x,
$$

and where the micro solutions $u_{j}^{h}\left(\right.$ resp. $\left.w_{j}^{h}\right)$ satisfy $u_{j}^{h}-u_{\operatorname{lin}, j}^{H} \in V^{q}\left(K_{\delta_{j}}, \mathcal{T}_{h}\right)$ and

$$
\int_{K_{\delta_{j}}} a^{\varepsilon}(x) e\left(u_{j}^{h}\right): e\left(z^{h}\right) \mathrm{d} x=0, \quad \forall z^{h} \in V^{q}\left(K_{\delta_{j}}, \mathcal{T}_{h}\right) .
$$

The term $u_{\operatorname{lin}, j}^{H}(x)$ corresponds to a linearization of $u^{H}$ at the integration nodes $x_{j, K}$, i.e.,

$$
u_{\operatorname{lin}, j}^{H}(x)=u^{H}\left(x_{j, K}\right)+\left(x-x_{j, K}\right) e\left(u^{H}\left(x_{j, K}\right)\right) .
$$


Note that the micro solutions $u_{j}^{h}$ do not depend on time and that the micro problems (14) are well-posed, which follows from the Lax-Milgram lemma together with Korn's inequality. As a consequence, problem (12) is well-posed and, in particular, admits a unique solution $u^{H} \in L^{\infty}\left(0, T ; V_{0}^{p}\left(\Omega, \mathcal{T}_{H}\right)\right)$, for all $\varepsilon, H, h>0$ (see $\left.[6,7,19]\right)$.

\section{Recovery of the homogenized tensor}

A numerical approximation of the homogenized tensor $a^{0}$ can be obtained during the assembly process of the FE-HMM. For general symmetric tensors and sampling domains, we define at each quadrature node $x_{j, K}$, two tensors $a_{K}^{0}$ and $\bar{a}_{K}^{0}$ by

$$
\left(a_{K}^{0}\left(x_{j, K}\right)\right)_{i k l m}=\frac{1}{\left|K_{\delta_{j}}\right|} \int_{K_{\delta_{j}}} a^{\varepsilon}(x) e\left(\varphi_{j i k}^{h}\right): e\left(\varphi_{j l m}^{h}\right) \mathrm{d} x
$$

and

$$
\left(\bar{a}_{K}^{0}\left(x_{j, K}\right)\right)_{i k l m}=\frac{1}{\left|K_{\delta_{j}}\right|} \int_{K_{\delta_{j}}} a^{\varepsilon}(x) e\left(\varphi_{j i k}\right): e\left(\varphi_{j l m}\right) \mathrm{d} x
$$

where $\varphi_{j i k}^{h} \in V^{q}\left(K_{\delta_{j}}, \mathcal{T}_{h}\right)\left(\varphi_{j i k} \in W\left(K_{\delta_{j}}\right)\right)$ are the solutions of $(14)$ and $i, k, l, m=1, \ldots, d$.

\section{A priori error analysis}

In this section, we derive a priori estimates for the error between the homogenized solution $u^{0}$ of (8) and the FE-HMM solution $u^{H}$ of (12). The total error splits into three separate contributions: a macro error, which primarily depends on the macro mesh size $H$, a modeling error which measures the accuracy of the upscaling procedure, and a micro error mainly determined by the micro mesh size $h$. Hence the combined modeling and micro error, $e_{H M M}$, is bounded by its two separate contributions as

$$
e_{H M M}=\left\|a^{0}\left(x_{j, K}\right)-a_{K}^{0}\left(x_{j, K}\right)\right\|_{F} \leq\left\|a^{0}\left(x_{j, K}\right)-\bar{a}_{K}^{0}\left(x_{j, K}\right)\right\|_{F}+\left\|\bar{a}_{K}^{0}\left(x_{j, K}\right)-a_{K}^{0}\left(x_{j, K}\right)\right\|_{F},
$$

with

$$
\begin{aligned}
e_{M O D} & =\left\|a^{0}\left(x_{j, K}\right)-\bar{a}_{K}^{0}\left(x_{j, K}\right)\right\|_{F}, \\
e_{M I C} & =\left\|\bar{a}_{K}^{0}\left(x_{j, K}\right)-a_{K}^{0}\left(x_{j, K}\right)\right\|_{F},
\end{aligned}
$$

and where $a_{K}^{0}$ and $\bar{a}_{K}^{0}$ are defined in (15) and (16) respectively.

The micro error $e_{M I C}$ results from the FE discretization of the micro problems and can be bounded without any assumptions about the spatial structure of the tensor $a^{\varepsilon}$ apart from (4)-(6). If the solutions $\psi^{i} \in W\left(K_{\delta_{j}}\right)$ of the continuous counterpart of (14) in fact are in $H^{q+1}\left(K_{\delta_{j}}\right)^{d}$ and satisfy

$$
\left|\psi^{i}\right|_{H^{q+1}\left(K_{\delta_{j}}\right)} \leq C \varepsilon^{-q}\left|K_{\delta_{j}}\right|^{1 / 2}, \text { for all } K \in \mathcal{T}_{H}, i=1, \ldots, d,
$$


then it holds for each macro element $K \in \mathcal{T}_{H}([1,2,9])$ :

$$
e_{M I C} \leq C\left(\frac{h}{\varepsilon}\right)^{2 q} .
$$

To determine an upper bound on the modeling error $e_{M O D}$, we need to make additional assumptions about the tensor $a^{\varepsilon}$, such as local periodicity $([2,9])$; i.e.,

(H3) $a^{\varepsilon}(x)=a(x, x / \varepsilon)=a(x, y)$ is $Y$-periodic in $y$.

If $a^{\varepsilon}$ satisfies (H3) with $a_{i j k l}(x, y) \in W^{1, \infty}\left(\bar{\Omega}, L^{\infty}(Y)\right)$ for all $i, j, k, l=1, \ldots, d$, we have

$$
\begin{array}{lll}
e_{M O D} \leq C \varepsilon, & \text { if } W\left(K_{\delta_{j}}\right)=H_{\mathrm{per}}^{1}\left(K_{\delta_{j}}\right)^{d}, & \delta / \varepsilon \in \mathbb{N}, \\
e_{M O D} \leq C\left(\delta+\frac{\varepsilon}{\delta}\right), & \text { if } W\left(K_{\delta_{j}}\right)=H_{0}^{1}\left(K_{\delta_{j}}\right)^{d}, & \delta / \varepsilon \notin \mathbb{N}, \delta>\varepsilon .
\end{array}
$$

Furthermore, if we collocate the slow variable $x$ in $a^{\varepsilon}$ at the quadrature points $x_{j, K}$ in the evaluation of the macro (13) and micro (14) bilinear forms, the modeling error reduces to

$$
\begin{array}{lll}
e_{M O D}=0, & \text { if } W\left(K_{\delta_{j}}\right)=H_{\mathrm{per}}^{1}\left(K_{\delta_{j}}\right)^{d}, & \delta / \varepsilon \in \mathbb{N}, \\
e_{M O D} \leq C \frac{\varepsilon}{\delta}, & \text { if } W\left(K_{\delta_{j}}\right)=H_{0}^{1}\left(K_{\delta_{j}}\right)^{d}, & \delta / \varepsilon \notin \mathbb{N}, \delta>\varepsilon .
\end{array}
$$

To derive an a priori error analysis, we now consider the elliptic projection $\pi_{H} u^{0}$ of the homogenized solution $u^{0}$, defined by

$$
B_{H}\left(\pi_{H} u^{0}, v^{H}\right)=B^{0}\left(u^{0}, v^{H}\right)+\left(\partial_{t t} u^{0}, v^{H}\right)-\left(I_{H} \partial_{t t} u^{0}, v^{H}\right)_{\mathrm{H}},
$$

where $I_{H}$ is any nodal interpolant which satisfies

$$
\left\|v-I_{H} v\right\|_{\mathrm{H}^{m}(\Omega)} \leq C H^{k-m}\|v\|_{\mathrm{H}^{k}(\Omega)}, \quad 0 \leq m \leq 1, \quad 2 \leq k \leq p+1 .
$$

Since $I_{H} u^{0} \in V_{0}^{p}\left(\Omega, \mathcal{T}_{H}\right)$ and $B_{H}$ is coercive, the elliptic projection $\pi_{H} u^{0} \in V_{0}^{p}\left(\Omega, \mathcal{T}_{H}\right)$ is well-defined. We can define the initial conditions of (12) as

$$
u^{H}(0)=I_{H} u^{0}(0)=I_{H} g_{1}, \quad \text { and } \quad \partial u^{H}(0)=I_{H} \partial_{t} u^{0}(0)=I_{H} g_{2} .
$$

To derive an upper bound for

$$
\left\|\partial_{t}\left(u^{0}-u^{H}\right)\right\|_{L^{\infty}\left(0, T ; L^{2}(\Omega)\right)}+\left\|u^{0}-u^{H}\right\|_{L^{\infty}\left(0, T ; H^{1}(\Omega)\right)},
$$

we shall estimate the difference between $\pi_{H} \partial_{t}^{\nu} u^{0}$ and $\partial_{t}^{\nu} u^{0}$ for $k=0,1,2$. To do so, we first recall the following estimates (given in [17] for simplicial elements), which hold for $v^{H}, w^{H} \in V^{p}\left(\Omega, \mathcal{T}_{H}\right)$ and $m=0,1:$

$$
\begin{aligned}
\left|B^{0}\left(v^{H}, w^{H}\right)-B_{H}^{0}\left(v^{H}, w^{H}\right)\right| & \leq C H^{p+m} \max _{i, j, k, l}\left\|a_{i j k l}^{0}\right\|_{\mathrm{W}^{p+m, \infty}(\Omega)}\left\|v^{H}\right\|_{\overline{\mathrm{H}}^{p+m}(\Omega)}\left\|w^{H}\right\|_{\overline{\mathrm{H}}^{1+m}(\Omega)}, \\
\left|\left(v^{H}, w^{H}\right)-\left(v^{H}, w^{H}\right)_{\mathrm{H}}\right| & \leq C H^{p+m}\left\|v^{H}\right\|_{\overline{\mathrm{H}}^{p+m}(\Omega)}\left\|w^{H}\right\|_{\overline{\mathrm{H}}^{1+m}(\Omega)},
\end{aligned}
$$


where $\|\cdot\|_{\overline{\mathrm{H}}^{p}(\Omega)}$ is a broken norm, given by

$$
\|v\|_{\overline{\mathrm{H}}^{p}(\Omega)}=\left(\sum_{K \in \mathcal{T}_{H}}\|v\|_{\mathrm{H}^{p}(K)}^{2}\right)^{1 / 2} .
$$

Similar estimates also hold for rectangular elements, see [18].

Lemma 4.1. Suppose that (22) and (23) holds for $m=0$ and that

$$
\begin{array}{ll}
\partial_{t}^{\nu} u^{0} \in L^{2}\left(0, T ; H^{p+1}(\Omega)\right), & \nu=0,1,2, \\
\partial_{t}^{2+\nu} u^{0} \in L^{2}\left(0, T ; H^{p}(\Omega)\right), & \nu=1,2 \\
a_{i j k l}^{0} \in W^{p, \infty}(\Omega), & i, j, k, l=1, \ldots, d .
\end{array}
$$

Then

$$
\left\|\partial_{t}^{\nu} u^{0}-\pi_{H} \partial_{t}^{\nu} u^{0}\right\|_{L^{2}\left(0, T ; \mathrm{H}^{1}(\Omega)\right)} \leq C\left(H^{p}+e_{M I C}+e_{M O D}\right),
$$

where the constant $C$ is independent of $H, h$, and $\varepsilon$. The micro error $e_{M I C}$ and the modeling error $e_{M O D}$ are given by (17) and (18) or (19), respectively.

Proof. We give the proof for $\nu=0$. For higher $\nu$, the proof follows by differentiating (20). Let $B_{H}^{0}$ denote the bilinear form corresponding to a standard FE discretization applied to (8),

$$
B_{H}^{0}\left(v^{H}, w^{H}\right)=\sum_{K \in \mathcal{T}_{H}} \sum_{j=1}^{J} \omega_{j, K} a^{0}\left(x_{j, K}\right) e\left(v^{H}\left(x_{j, K}\right)\right) e\left(w^{H}\left(x_{j, K}\right)\right) .
$$

By definition of the elliptic projection (20), we have

$$
\begin{aligned}
B_{H}\left(\pi_{H} u^{0}-I_{H} u^{0}, v^{H}\right)= & B^{0}\left(u^{0}-I_{H} u^{0}, v^{H}\right)+B_{0}\left(I_{H} u^{0}, v^{H}\right)-B_{H}\left(I_{H} u^{0}, v^{H}\right)+\left(\partial_{t t} u^{0}, v^{H}\right) \\
& -\left(I_{H} \partial_{t t} u^{0}, v^{H}\right)_{\mathrm{H}}+\left(I_{H} \partial_{t t} u^{0}, v^{H}\right)-\left(I_{H} \partial_{t t} u^{0}, v^{H}\right) \\
= & B^{0}\left(u^{0}-I_{H} u^{0}, v^{H}\right)+B^{0}\left(I_{H} u^{0}, v^{H}\right)-B_{H}^{0}\left(I_{H} u^{0}, v^{H}\right) \\
& +B_{H}^{0}\left(I_{H} u^{0}, v^{H}\right)-B_{H}\left(I_{H} u^{0}, v^{H}\right) \\
& +\left(\partial_{t t} u^{0}-I_{H} \partial_{t t} u^{0}, v^{H}\right)+\left(I_{H} \partial_{t t} u^{0}, v^{H}\right)-\left(I_{H} \partial_{t t} u^{0}, v^{H}\right)_{\mathrm{H} .} .
\end{aligned}
$$

We bound each term and use the short-hand notation $\|\cdot\|_{L^{2}\left(H^{p}\right)}$ to denote the norm $\|\cdot\|_{\mathrm{L}^{2}\left(0, T ; H^{p}(\Omega)\right)}, p \geq 1$. Using the boundedness of $B^{0}$, it holds

$$
B^{0}\left(u^{0}-I_{H} u^{0}, v^{H}\right) \leq C H^{p}\left\|u^{0}\right\|_{L^{2}\left(H^{p+1}\right)}\left\|v^{H}\right\|_{L^{2}\left(H^{1}\right)},
$$

where $I_{H}$ satisfies (21). For the second and third terms, we use equation (22) with $m=0$ to obtain

$$
B^{0}\left(I_{H} u^{0}, v^{H}\right)-B_{H}^{0}\left(I_{H} u^{0}, v^{H}\right) \leq C H^{p}\left\|I_{H} u^{0}\right\|_{L^{2}\left(\bar{H}^{p}\right)}\left\|v^{H}\right\|_{L^{2}\left(H^{1}\right)} \leq C H^{p}\left\|u^{0}\right\|_{L^{2}\left(H^{p+1}\right)}\left\|v^{H}\right\|_{L^{2}\left(H^{1}\right)} .
$$


From [1, Lemma 4.3] and [7, Lemma 4.1], it then holds

$B_{H}^{0}\left(I_{H} u^{0}, v^{H}\right)-B_{H}\left(I_{H} u^{0}, v^{H}\right) \leq C e_{H M M}\left\|I_{H} u^{0}\right\|_{L^{2}\left(H^{1}\right)}\left\|v^{H}\right\|_{L^{2}\left(H^{1}\right)} \leq C e_{H M M}\left\|u^{0}\right\|_{L^{2}\left(H^{1}\right)}\left\|v^{H}\right\|_{L^{2}\left(H^{1}\right)}$.

We bound the first inner product by

$$
\left(\partial_{t t} u^{0}-I_{H} \partial_{t t} u^{0}, v^{H}\right) \leq C H^{p}\left\|\partial_{t t} u^{0}\right\|_{L^{2}\left(H^{p}\right)}\left\|v^{H}\right\|_{L^{2}\left(H^{1}\right)},
$$

where we have used estimate (21). The last term is bounded by

$$
\left(I_{H} \partial_{t t} u^{0}, v^{H}\right)-\left(I_{H} \partial_{t t} u^{0}, v^{H}\right)_{\mathrm{H}} \leq C H^{p}\left\|u^{0}\right\|_{L^{2}\left(H^{p}\right)}\left\|v^{H}\right\|_{L^{2}\left(H^{1}\right)} .
$$

By using the coercivity of $B_{H}$, we now obtain

$$
\begin{aligned}
\left\|\pi_{H} u^{0}-I_{H} u^{0}\right\|_{L^{2}\left(H^{1}\right)}^{2} & \leq C B_{H}\left(\pi_{H} u^{0}-I_{H} u^{0}, \pi_{H} u^{0}-I_{H} u^{0}\right) \\
& \leq C\left(H^{p}\left\|u^{0}\right\|_{L^{2}\left(H^{p}\right)}+e_{H M M}\left\|u^{0}\right\|_{L^{2}\left(H^{1}\right)}+H^{p}\left\|\partial_{t t} u^{0}\right\|_{L^{2}\left(H^{p}\right)}\right)\left\|\pi_{H} u^{0}-I_{H} u^{0}\right\|_{L^{2}\left(H^{1}\right)} .
\end{aligned}
$$

To conclude we use integration in time, estimate (21), and the fact that $e_{H M M} \leq e_{M I C}+e_{M O D}$ together with the triangle inequality

$$
\left\|u^{0}-\pi_{H} u^{0}\right\|_{L^{2}\left(H^{1}\right)} \leq\left\|u^{0}-I_{H} u^{0}\right\|_{L^{2}\left(H^{1}\right)}+\left\|\pi_{H} u^{0}-I_{H} u^{0}\right\|_{L^{2}\left(H^{1}\right)} .
$$

A similar Lemma holds for the $L^{2}$ norm, where we combine a classical Aubin-Nitsche argument with the $H^{1}$ estimates given in Lemma 4.1.

Lemma 4.2. Suppose that (22) and (23) hold for $m=1$ and that

$$
\begin{array}{ll}
\partial_{t}^{\nu} u^{0} \in L^{2}\left(0, T ; H^{p+1}(\Omega)\right), & \nu=0,1, \\
\partial_{t}^{2+\nu} u^{0} \in L^{2}\left(0, T ; H^{p}(\Omega)\right), & \nu=0,1, \\
a_{i j k l}^{0} \in W^{p+1, \infty}(\Omega), & i, j, k, l=1, \ldots, d .
\end{array}
$$

Then

$$
\left\|\partial_{t}^{\nu} u^{0}-\pi_{H} \partial_{t}^{\nu} u^{0}\right\|_{\mathrm{L}^{2}\left(0, T ; \mathrm{L}^{2}(\Omega)\right)} \leq C\left(H^{p+1}+e_{M I C}+e_{M O D}\right)
$$

where the constant $C$ is independent of $H, h$, and $\varepsilon$. The micro error $e_{M I C}$ and the modeling $e_{M O D}$ are given by (17) and (18) or (19), respectively.

We can now express a bound for the difference between $u^{0}$ and $u^{H}$.

Theorem 4.3. Let $u^{0}$ and $u^{H}$ be the solutions of (8) and (12), respectively. Suppose that (22) and (23) hold for $m=0$. Further assume that (21) holds and that

$$
\begin{array}{ll}
\partial_{t}^{\nu} u^{0} \in L^{2}\left(0, T ; H^{p+1}(\Omega)\right), & \nu=0,1,2, \\
\partial_{t}^{2+\nu} u^{0} \in L^{2}\left(0, T ; H^{p}(\Omega)\right), & \nu=1,2, \\
a_{i j k l}^{0} \in W^{p, \infty}(\Omega), & i, j, k, l=1, \ldots, d, \\
g_{1} \in\left(H^{p+1}(\Omega)\right)^{d}, \quad g_{2} \in\left(H^{\max (2, p)}(\Omega)\right)^{d}, & \\
\partial_{t}^{\nu} u^{H} \in L^{2}\left(0, T ; H^{1}(\Omega)\right), \partial_{t}^{2} u^{H} \in L^{2}\left(0, T ; L^{2}(\Omega)\right) & \nu=0,1 .
\end{array}
$$


Then

$$
\left\|\partial_{t}\left(u^{0}-u^{H}\right)\right\|_{L^{\infty}\left(0, T ; \mathrm{L}^{2}(\Omega)\right)}+\left\|u^{0}-u^{H}\right\|_{\mathrm{L}^{\infty}\left(0, T ; \mathrm{H}^{1}(\Omega)\right)} \leq C\left(H^{p}+e_{M I C}+e_{M O D}\right),
$$

where the constant $C$ is independent of $H, h$, and $\varepsilon$. The micro error $e_{M I C}$ and the modeling error $e_{M O D}$ are given by (17) and (18) or (19), respectively.

Proof. Here we use the short-hand notation $\|\cdot\|_{L^{2}\left(H^{p}\right)}$ to denote the norm $\|\cdot\|_{\mathrm{L}^{2}\left(0, T ; H^{p}(\Omega)\right)}$, $p \geq 1$. First, we decompose the error for $u^{0}-u^{H}$ as

$$
\left\|u^{0}-u^{H}\right\|_{L^{2}\left(H^{1}\right)} \leq\left\|u^{0}-\pi_{H} u^{0}\right\|_{L^{2}\left(H^{1}\right)}+\left\|\pi_{H} u^{0}-u^{H}\right\|_{L^{2}\left(H^{1}\right)} .
$$

The first error term is bounded according to Lemma 4.1 as

$$
\left\|u^{0}-\pi_{H} u^{0}\right\|_{L^{2}\left(H^{1}\right)} \leq C\left(H^{p}+e_{H M M}\right) .
$$

Next, we compute

$$
\begin{aligned}
& \left(\partial_{t t}\left(u^{H}-\pi_{H} u^{0}\right), v^{H}\right)_{\mathrm{H}}+B_{H}\left(u^{H}-\pi_{H} u^{0}, v^{H}\right) \\
& =\left(\partial_{t t} u^{H}, v^{H}\right)_{\mathrm{H}}-\left(\partial_{t t} \pi_{H} u^{0}, v^{H}\right)_{\mathrm{H}}+B_{H}\left(u^{H}, v^{H}\right)-B_{H}\left(\pi_{H} u^{0}, v^{H}\right) \\
& =F\left(v^{H}\right)-\left(\partial_{t t} \pi_{H} u^{0}, v^{H}\right)_{\mathrm{H}}-B_{H}\left(\pi_{H} u^{0}, v^{H}\right) \\
& =\left(\partial_{t t} u^{0}, v^{H}\right)_{\mathrm{H}}+B^{0}\left(u^{0}, v^{H}\right)-\left(\partial_{t t} \pi_{H} u^{0}, v^{H}\right)_{\mathrm{H}}-B_{H}\left(\pi_{H} u^{0}, v^{H}\right) \\
& =\left(\partial_{t t} u^{0}, v^{H}\right)_{\mathrm{H}}+B^{0}\left(u^{0}, v^{H}\right)-\left(\partial_{t t} \pi_{H} u^{0}, v^{H}\right)_{\mathrm{H}}-B^{0}\left(u^{0}, v^{H}\right)-\left(\partial_{t t} u^{0}, v^{H}\right)_{\mathrm{H}}+\left(I_{H} \partial_{t t} u^{0}, v^{H}\right)_{\mathrm{H}} \\
& =\left(I_{H} \partial_{t t} u^{0}-\partial_{t t} \pi_{H} u^{0}, v^{H}\right)_{\mathrm{H}},
\end{aligned}
$$

where we have used (20) to replace $B_{H}\left(\pi_{H} u^{0}, v^{H}\right)$.

Now, let $\eta_{H}=\left(u^{H}-\pi_{H} u^{0}\right)$ and note that

$$
\frac{1}{2} \frac{d}{d t}\left(\left(\partial_{t} \eta_{H}, \partial_{t} \eta_{H}\right)_{\mathrm{H}}+B_{H}\left(\eta_{H}, \eta_{H}\right)\right)=\left(I_{H} \partial_{t t} u^{0}-\pi_{H} \partial_{t t} u^{0}, \partial_{t} \eta_{H}\right)_{\mathrm{H}}
$$

With $\xi(t)=\left(\partial_{t} \eta_{H}, \partial_{t} \eta_{H}\right)_{\mathrm{H}}+B_{H}\left(\eta_{H}, \eta_{H}\right)$, we obtain from Young's inequality

$$
\frac{1}{2} \frac{d}{d t} \xi(t) \leq C\left(\left\|I_{H} \partial_{t t} u^{0}-\pi_{H} \partial_{t t} u^{0}\right\|_{L^{2}\left(L^{2}\right)}^{2}+\left\|\partial_{t} \eta_{H}\right\|_{L^{2}\left(L^{2}\right)}^{2}\right) .
$$

Since

$$
\left\|\partial_{t} \eta_{H}\right\|_{L^{2}\left(L^{2}\right)}^{2} \leq\left(\partial_{t} \eta_{H}, \partial_{t} \eta_{H}\right)_{\mathrm{H}} \leq\left(\partial_{t} \eta_{H}, \partial_{t} \eta_{H}\right)_{\mathrm{H}}+B_{H}\left(\eta_{H}, \eta_{H}\right)=\xi(t)
$$

it holds

$$
\frac{1}{2} \frac{d}{d t} \xi(t) \leq C\left(\left\|I_{H} \partial_{t t} u^{0}-\pi_{H} \partial_{t t} u^{0}\right\|_{L^{2}\left(L^{2}\right)}^{2}+\xi(t)\right) .
$$

From Gronwall's inequality, we thus obtain the upper bound

$$
\sup _{0 \leq t \leq T} \xi(t) \leq C\left(\xi(0)+\left\|I_{H} \partial_{t t} u^{0}-\pi_{H} \partial_{t t} u^{0}\right\|_{L^{2}\left(L^{2}\right)}^{2}\right),
$$


which implies

$$
\sup _{0 \leq t \leq T} \xi(t) \leq C\left(\xi(0)+H^{2(p+1)}+e_{H M M}^{2}\right)
$$

by using Lemma 4.2 .

It remains to bound $\xi(0)$. By definition, we have

$$
\xi(0)=\left.\left(\left(\partial_{t} \eta_{H}, \partial_{t} \eta_{H}\right)_{\mathrm{H}}+B_{H}\left(\eta_{H}, \eta_{H}\right)\right)\right|_{t=0} \leq C\left(\left\|\partial_{t} \eta_{H}(0)\right\|_{\mathrm{L}^{2}(\Omega)}^{2}+B_{H}\left(\eta_{H}(0), \eta_{H}(0)\right) .\right.
$$

Since $u^{0}(0)=g_{1}$, we obtain that

$$
\begin{aligned}
\left|B_{H}\left(\eta_{H}(0), \eta_{H}(0)\right)\right| & \leq C\left\|\eta_{H}(0)\right\|_{\mathrm{H}^{1}(\Omega)}^{2}=C\left\|u^{H}(0)-\pi_{H} u^{0}(0)\right\|_{\mathrm{H}^{1}(\Omega)}^{2} \\
& =C\left\|I_{H} g_{1}-\pi_{H} u^{0}(0)\right\|_{\mathrm{H}^{1}(\Omega)}^{2} \\
& \leq C\left(\left\|I_{H} g_{1}-g_{1}\right\|_{\mathrm{H}^{1}(\Omega)}^{2}+\left\|u^{0}(0)-\pi_{H} u^{0}(0)\right\|_{\mathrm{H}^{1}(\Omega)}^{2}\right) \\
& \leq C\left(H^{2 p}\left\|g_{1}\right\|_{\mathrm{H}^{p+1}(\Omega)}^{2}+\left\|u^{0}-\pi_{H} u^{0}\right\|_{L^{2}\left(H^{1}\right)}^{2}+\left\|\partial_{t} u^{0}-\pi_{H} \partial_{t} u^{0}\right\|_{L^{2}\left(H^{1}\right)}^{2}\right) \\
& \leq C\left(H^{2 p}\left\|g_{1}\right\|_{\mathrm{H}^{p+1}(\Omega)}^{2 p}+H^{2 p}+e_{H M M}^{2}\right),
\end{aligned}
$$

where we have used the continuous embedding of $H^{1}(\Omega)$ into $\mathcal{C}(\Omega)$, Lemma 4.1, estimate (21) with $m=1, k=p+1$, and the regularity assumption on $g_{1}$. Similarly,

$$
\begin{aligned}
\left\|\partial_{t} \eta_{H}(0)\right\|_{\mathrm{L}^{2}(\Omega)} & =\left\|I_{H} g_{2}-\pi_{H} \partial_{t} u^{0}(0)\right\|_{\mathrm{L}^{2}(\Omega)} \\
& \leq C\left(\left\|I_{H} g_{2}-g_{2}\right\|_{\mathrm{L}^{2}(\Omega)}+\left\|\partial_{t} u^{0}(0)-\pi_{H} \partial_{t} u^{0}(0)\right\|_{\mathrm{L}^{2}(\Omega)}\right) \\
& \leq C\left(H^{p}\left\|g_{2}\right\|_{\mathrm{H}^{p}(\Omega)}+\left\|\partial_{t} u^{0}-\pi_{H} \partial_{t} u^{0}\right\|_{L^{2}\left(L^{2}\right)}+\left\|\partial_{t t} u^{0}-\pi_{H} \partial_{t t} u^{0}\right\|_{L^{2}\left(L^{2}\right)}\right) \\
& \leq C\left(H^{p}\left\|g_{2}\right\|_{\mathrm{H}^{p}(\Omega)}+H^{p+1}+e_{H M M}\right) .
\end{aligned}
$$

Combining the above estimates, we thus obtain

$$
\sup _{0 \leq t \leq T} \xi(t) \leq C\left(H^{2 p}+e_{H M M}^{2}\right)
$$

and

$$
\left\|\partial_{t} \eta_{H}\right\|_{L^{\infty}\left(L^{2}\right)}^{2}+\left\|\eta_{H}\right\|_{L^{\infty}\left(H^{1}\right)}^{2} \leq C \sup _{0 \leq t \leq T} \xi(t) \leq C\left(H^{2 p}+e_{H M M}^{2}\right),
$$

which concludes the proof as $e_{H M M} \leq e_{M I C}+e_{M O D}$.

A priori error estimates also hold for the $L^{2}$ norm using a classical Aubin-Nitsche argument together with Lemmas 4.1 and 4.2 .

Theorem 4.4. Let $u^{0} \in H_{0}^{1}(\Omega)^{d}$ and $u^{H}$ be solution of (8) and (12) respectively. Suppose that (22) and (23) hold for $m=1$. Further assume that (21) holds and

$$
\begin{array}{ll}
\partial_{t}^{\nu} u^{0} \in L^{2}\left(0, T ; H^{p+1}(\Omega)\right), & \nu=0,1,2,3 \\
a_{i j k l}^{0} \in W^{p+1, \infty}(\Omega), & i, j, k, m, l=1, \ldots, d, \\
g_{1} \in H^{p+1}(\Omega)^{d}, & \\
\partial_{t}^{\nu} u^{H} \in L^{2}\left(0, T ; L^{2}(\Omega)\right), & \nu=0,1,
\end{array}
$$


Then

$$
\left\|u^{0}-u^{H}\right\|_{L^{\infty}\left(0, T ; \mathrm{L}^{2}(\Omega)\right)} \leq C\left(H^{p+1}+e_{M I C}+e_{M O D}\right),
$$

where the constant is independent of $H, h$, and $\varepsilon$. The micro $e_{M I C}$ and modeling $e_{M O D}$ errors are given by (17) and (18) or (19), respectively.

DOF for the FE-HMM

Optimal convergence rates can be obtained by balancing the macro and micro errors as follows:

$$
H^{p} \sim\left(\frac{h}{\varepsilon}\right)^{2 q}, \text { for the } H^{1} \text { norm; } H^{p+1} \sim\left(\frac{h}{\varepsilon}\right)^{2 q} \text { for the } L^{2} \text { norm. }
$$

Let $N_{\text {macro }}$ and $N_{\text {micro }}$ denote the total number of degrees of freedom used in the macro and micro solvers respectively, and assume that $H \sim \frac{1}{N_{\text {macro }}}$ and $\frac{h}{\varepsilon} \sim \frac{1}{N_{\text {micro }}}$. From the convergence rates given in Theorems 4.3 and 4.4, we thus obtain

$$
N_{\text {micro }} \sim N_{\text {macro }}^{p / 2 q}, \text { for the } H^{1} \text { norm; } \quad N_{\text {micro }}=N_{\text {macro }}^{(p+1) / 2 q}, \text { for the } L^{2} \text { norm. }
$$

\section{Numerical experiments}

In this section, we present a series of numerical experiments that illustrate the usefulness of the FE-HMM for wave propagation in a linear elastic media. We recall that we seek a numerical approximation of $u^{0}$, the solution of the homogenized elastic wave equation

$$
\partial_{t t} u^{0}-\operatorname{div}\left(a^{0}: e\left(u^{0}\right)\right)=f .
$$

In general, the expected a priori error rates, given in Theorems 4.3 and 4.4, are

$$
\begin{aligned}
\left\|u^{0}(T)-u^{H}(T)\right\|_{\mathrm{H}^{1}(\Omega)} & \leq C\left(H^{p}+e_{M I C}+e_{M O D}\right), \\
\left\|u^{0}(T)-u^{H}(T)\right\|_{L^{2}(\Omega)} & \leq C\left(H^{p+1}+e_{M I C}+e_{M O D}\right),
\end{aligned}
$$

where the micro $e_{M I C}$ and modeling $e_{M O D}$ errors are given in (17) and (18) (or (19) if collocation is used), respectively.

The tensors considered in the following two-dimensional experiments correspond to $4 \times 4$ matrices with entries $a_{i j k l}, i, j, k, l=1,2$. Due to symmetry, as in (4), only 9 entries are truly independent. Hence, we shall represent any heterogeneous tensor in the numerical experiments below by the $3 \times 3$ matrix

$$
a^{\varepsilon}(x)=\left(\begin{array}{lll}
a_{1111}^{\varepsilon} & a_{1122}^{\varepsilon} & a_{112}^{\varepsilon} \\
a_{1122}^{\varepsilon} & a_{2222}^{\varepsilon} & a_{2212}^{\varepsilon} \\
a_{1112}^{\varepsilon} & a_{2212}^{\varepsilon} & a_{1212}^{\varepsilon}
\end{array}\right) .
$$

First, we consider periodic or locally periodic tensors and verify the convergence rates of Theorems 4.3 and 4.4. Next, we consider a layered material and compare the FE-HMM results with those derived in [24] based on the MsFEM. Finally, we consider a layered random media, where the randomness is produced by a von Karman correlation function [26]. 


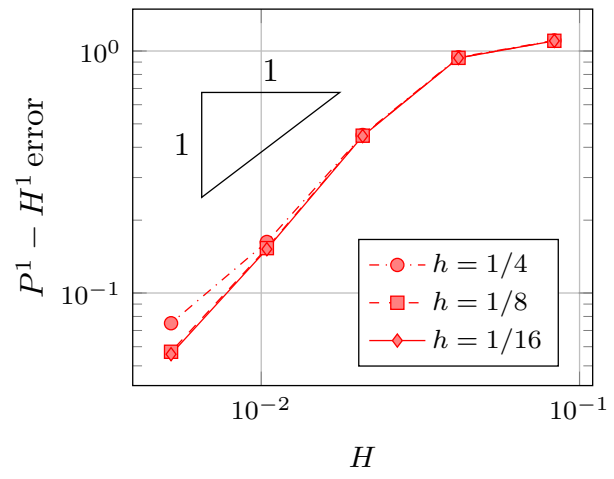

(a)

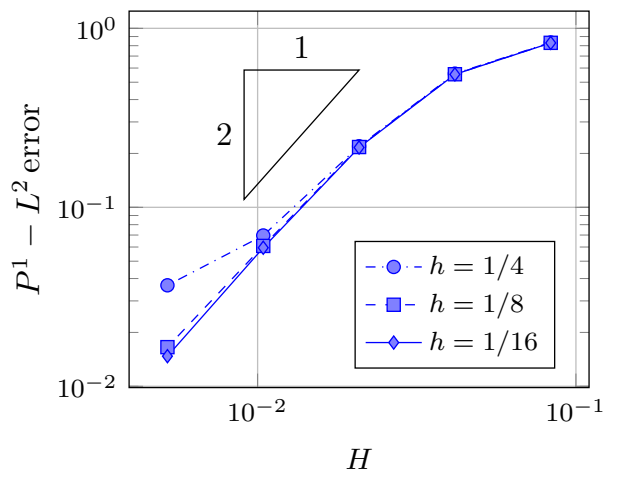

(b)

Figure 1: Periodic medium. Error $\left\|u^{0}(T)-u^{H}(T)\right\|$ vs. the macro mesh size $H$ for different fixed micro mesh sizes $h$ with $P^{1}$ macro and micro FE spaces: (a) $H^{1}(\Omega)$ and (b) $L^{2}(\Omega)$ norm.

\section{Periodic medium}

For our first experiment, we choose a $Y$-periodic tensor in $y$ and shall study convergence of $u^{0}$ and $u^{H}$ at time $T$. Let $\Omega=[-1,1]^{2}$ and $a^{0}$ be the homogenized tensor that corresponds to the heterogeneous tensor

$$
a^{\varepsilon}(x)=\left(\begin{array}{ccc}
\sin \left(2 \pi x_{1} / \varepsilon\right)+2 & 0 & 0 \\
0 & \sin \left(2 \pi x_{2} / \varepsilon\right)+2 & 0 \\
0 & 0 & 10
\end{array}\right) .
$$

Explicit equations are available (see $[13,34,19])$ for $a^{0}$, which is given by

$$
a^{0}=\left(\begin{array}{ccc}
\sqrt{3} & 0 & 0 \\
0 & \sqrt{3} & 0 \\
0 & 0 & 10
\end{array}\right)
$$

We choose homogeneous Neumann boundary conditions, a Gaussian initial condition $g_{1}$, and zero initial velocity $g_{2}$. Here, the reference solution $u^{0}$ is computed on a very fine uniform mesh with mesh size $h_{f}$. Collocation to the slow variable is used at the quadrature points, which reduces the modeling error to zero - see Section 4. Further, we set the size of the sampling domain to $\delta=\varepsilon$. Then, the micro error can be reformulated in terms of the number of degrees of freedom as $h / \varepsilon \approx 1 / N_{\text {micro }}$, whereas the convergence rates w.r.t. the $H^{1}$ and the $L^{2}$ norm are now given by

$$
\left\|u^{0}(T)-u^{H}(T)\right\|_{\mathrm{H}^{1}(\Omega)} \leq C\left(H^{p}+e_{M I C}\right), \quad\left\|u^{0}(T)-u^{H}(T)\right\|_{\mathrm{L}^{2}(\Omega)} \leq C\left(H^{p+1}+e_{M I C}\right) .
$$

To demonstrate that convergence can only be achieved when both the micro and the macro mesh are refined, we now proceed as follows: We consider a fixed micro mesh with mesh size $h$ sufficiently small so that the micro error remains negligible relative to the macro error at initial moderately fine macro mesh sizes $H$. As the macro mesh is progressively 


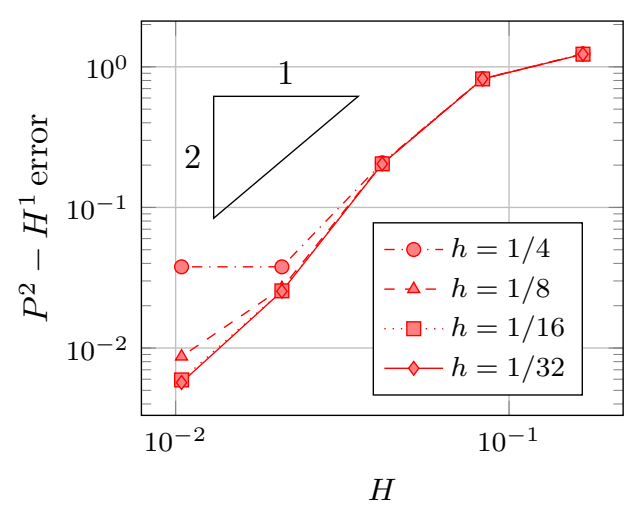

(a)

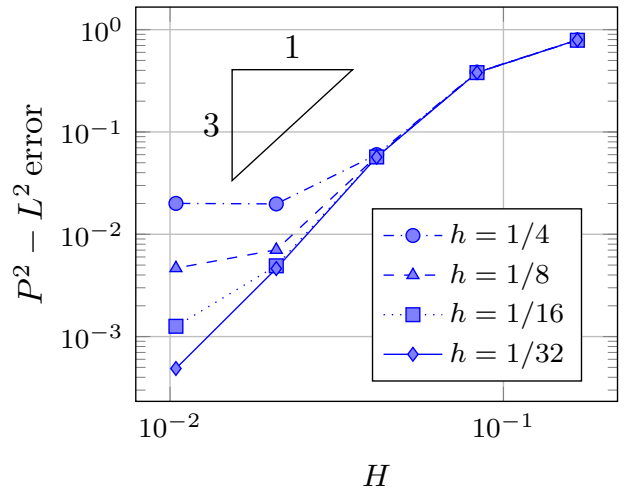

(b)

Figure 2: Periodic medium. Error $\left\|u^{0}(T)-u^{H}(T)\right\|$ vs. the macro mesh size $H$ for different fixed micro mesh sizes $h$ with $P^{2}$ macro and $P^{1}$ micro FE spaces: (a) $H^{1}(\Omega)$ and (b) $L^{2}(\Omega)$ norm.

refined, however, the macro error will eventually become smaller than the micro error and the convergence rates therefore stagnate. We set $\varepsilon=1 / 10, T=0.2$ and use an initial coarse mesh size of $H=1 / 12$. In Figures 1 (a) and 1(b), we consider piecewise linear macro and micro FE and display the $H^{1}$ and $L^{2}$ errors at time $t=0.2$ vs. the macro mesh size $H$, for different micro mesh sizes $h=\frac{1}{4}, \frac{1}{8}$, and $h=\frac{1}{16}$.

For time discretization, we always use the (explicit, second-order) leapfrog scheme and impose the CFL stability condition:

$$
\Delta t \leq \frac{h_{f}}{50}
$$

where $h_{f}$ is the mesh size of the fine mesh used to compute the reference solution. Similarly for the FE-HMM, we impose the CFL condition:

$$
(\Delta t)_{H M M} \leq \frac{H}{50} .
$$

Since $H$ is much larger than $h_{f}$, the CFL condition for the FE-HMM is much less restrictive than the CFL condition (24) used for the reference solutions $u^{0}$ and $u^{\varepsilon}$.

In Figures 2(a) and 2(b), we show the $H^{1}$ and $L^{2}$ errors vs. $H$ at time $t=0.2$, using $P^{2}$ macro and $P^{1}$ micro FE. The initial macro mesh is set to $H=1 / 6$ and the micro mesh sizes to $h=1 / 4,1 / 8,1 / 16$, and $h=1 / 32$. Here, optimal macro-micro refinements are given by

$$
N_{\text {micro }}=N_{\text {macro }}, \quad\left(H^{1} \text { norm }\right), \quad N_{\text {micro }}=N_{\text {macro }}^{3 / 2}, \quad\left(L^{2} \text { norm }\right) .
$$

Now the effect of the micro error in the $H^{1}$ and $L^{2}$ norm is even more apparent when $P^{2}$ macro FE are used with $P^{1}$ micro FE. Indeed, the micro error now is larger relatively to the macro error and hence the threshold value, where the micro error starts to dominate, more prominent.

Next, we display the horizontal cross-section at $x_{2}=0$ of the displacement in the $x_{1}$ direction and the horizontal cross-section at $x_{1}=0$ of the displacement in the $x_{2}$ direction 


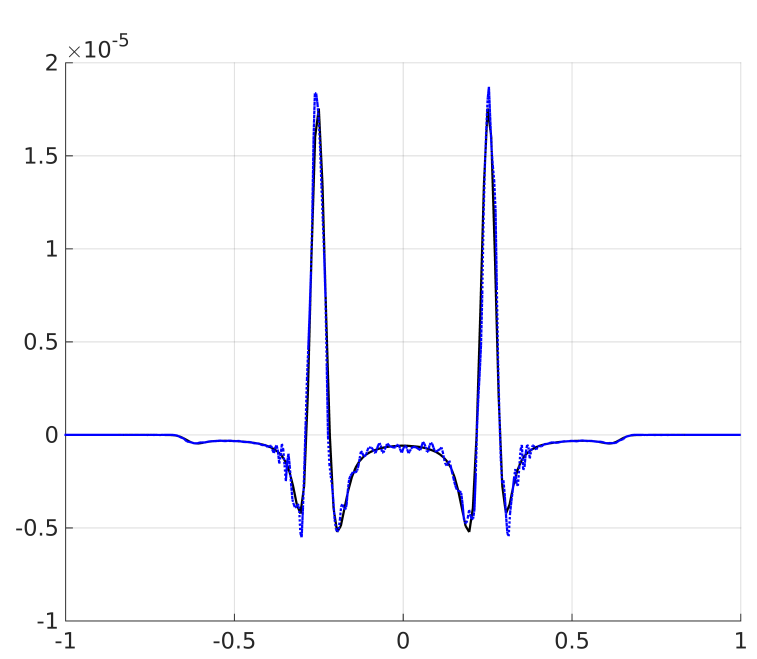

(a)

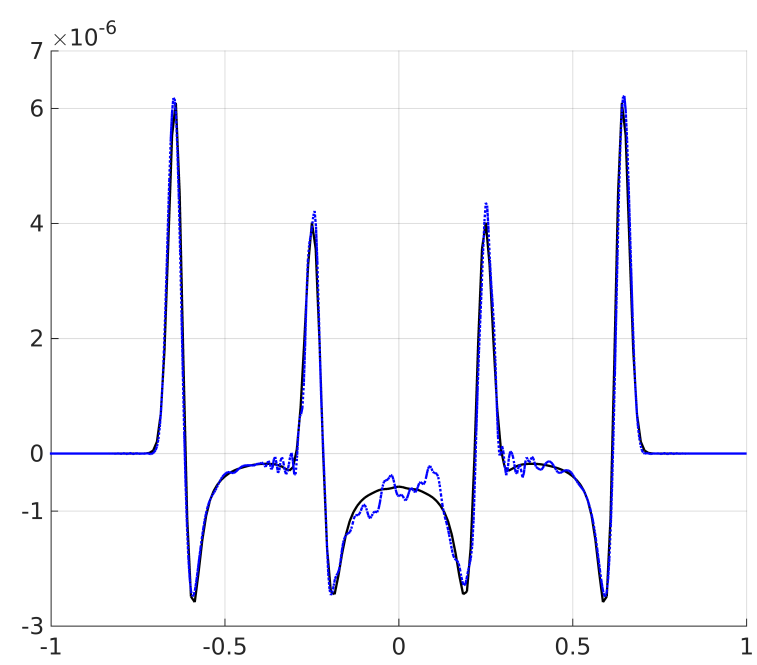

(b)

Figure 3: Periodic medium. Horizontal cross-sections (a) at $x_{2}=0$ in the $x_{1}$ direction and (b) at $x_{1}=0$ in the $x_{2}$ direction of the displacement fields $u^{0}$ (black) and $u^{\varepsilon}$ (dashed blue) at the final time $T=0.2$, with $\varepsilon=1 / 50$.

at the final time $T=0.2$ of $u^{0}$ and $u^{\varepsilon}$, computed using $P^{2}$ finite elements. In Figure 3(a), we show the amplitude of the displacement along the $x_{1}$ direction for the homogenized solution in black and for the fully resolved (true) solution with $\varepsilon=1 / 50$ in blue; the $x_{2}$ displacement is shown in Figure 3(b). In Figure 4, we compare the corresponding FE-HMM solution again with the homogenized solution $u^{0}$. Since the error between the two tensors $a_{K}^{0}$ and $a^{0}$ is small, $u^{0}$ and $u^{H}$ essentiall coincide.

As $\varepsilon$ diminishes, the homogenized solution captures increasingly better the global behavior of the heterogeneous solution. In Figures 5(a) and 5(b), we compare the two displacement fields along the $x_{1}$ and $x_{2}$ directions for $\varepsilon=1 / 100$, respectively.

\section{Locally periodic medium}

For our second experiment, we shall verify the convergence rates of Theorems 4.3 and 4.4 for a heterogeneous problem with a locally periodic tensor $a^{\varepsilon}$. Let $a^{\varepsilon}$ be defined by

$$
a^{\varepsilon}(x)=\left(\begin{array}{ccc}
\sin \left(2 \pi x_{1} / \varepsilon\right) \sin \left(x_{1}^{2} x_{2}^{2}\right)+2 & 0 & 0 \\
0 & \sin \left(2 \pi x_{2} / \varepsilon\right) \sin \left(x_{1}^{2} x_{2}^{2}\right)+2 & 0 \\
0 & 0 & 10
\end{array}\right) .
$$

Here, the exact homogenized tensor is not known and we use very fine meshes for the macro and micro problems. Collocation is still used at the quadrature points and we fix the micro mesh size sufficiently fine value to ensure that it is initially negligible relative to the micro error. We set $T=0.1$ and choose an initial mesh of size $H=1 / 8$. In Figures $6(\mathrm{a})$ and $6(\mathrm{~b})$, we show the $H^{1}$ and $L^{2}$ errors at the final time $T$, using $P^{2}$ macro and $P^{1}$ micro FE with sampling domains of different size $\delta$. 


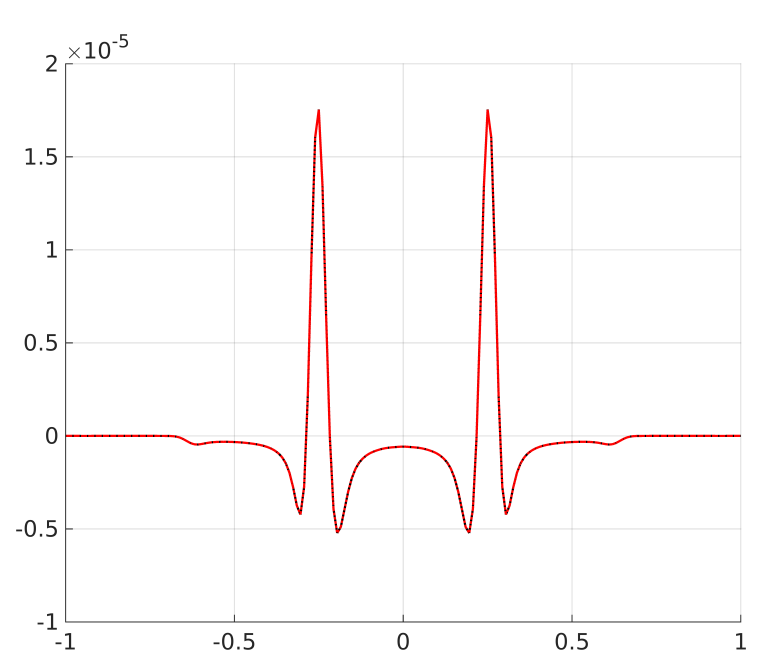

(a)

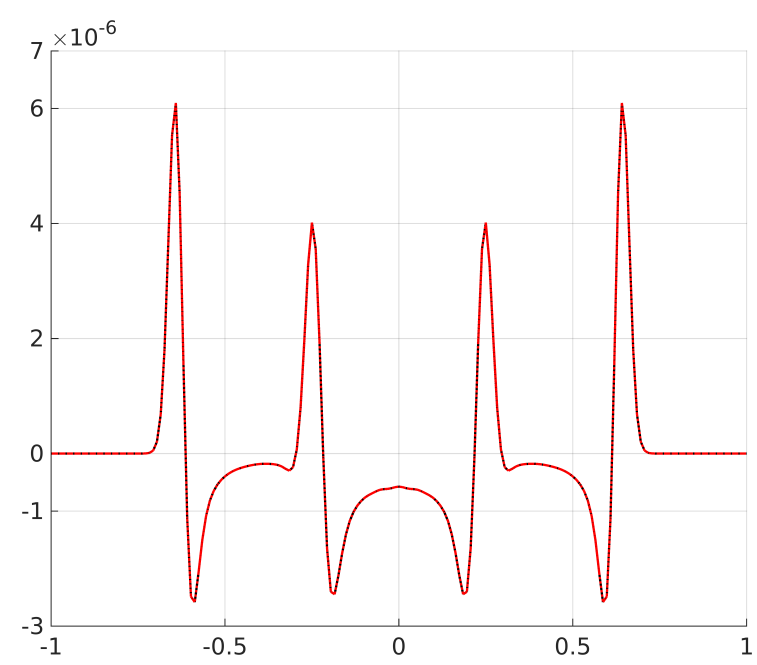

(b)

Figure 4: Periodic medium. Horizontal cross-sections (a) at $x_{2}=0$ in the $x_{1}$ direction and (b) at $x_{1}=0$ in the $x_{2}$ direction of the displacement fields $u^{0}$ (black) and $u^{H}$ (dashed red) at the final time $T=0.2$, with $\varepsilon=1 / 50$ and $h=1 / 32$.

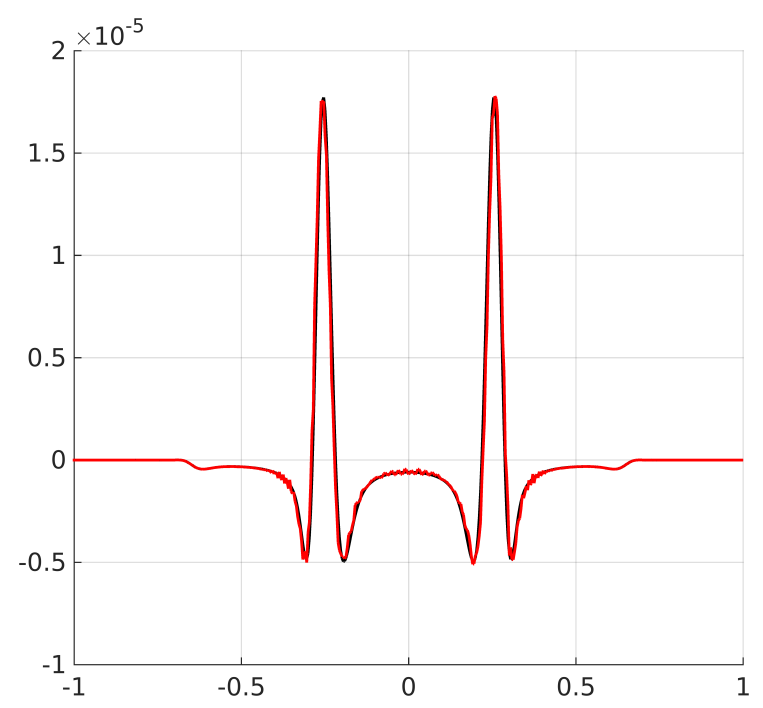

(a)

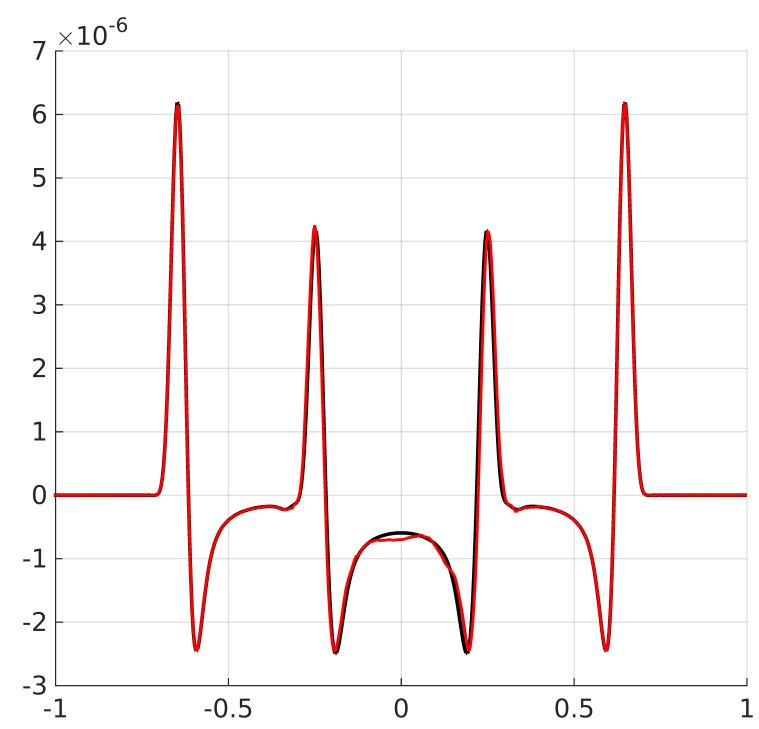

(b)

Figure 5: Periodic medium. Horizontal cross-sections (a) at $x_{2}=0$ in the $x_{1}$ direction and (b) at $x_{1}=0$ in the $x_{2}$ direction of the displacement fields $u^{0}$ (black) and $u^{\varepsilon}$ (red) at the final time $T=0.2$, with $\varepsilon=1 / 100$. 


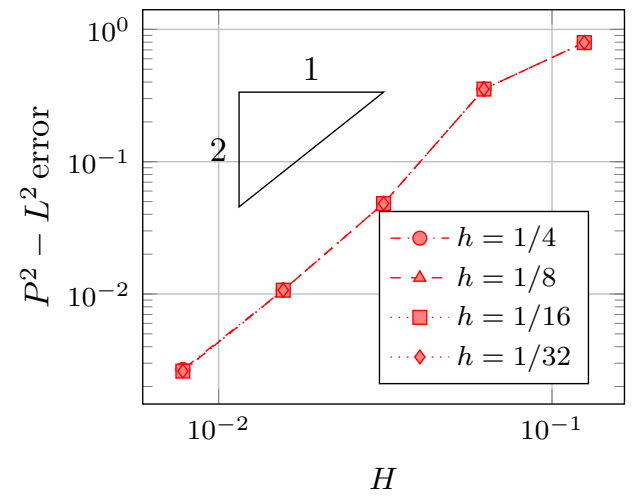

(a)

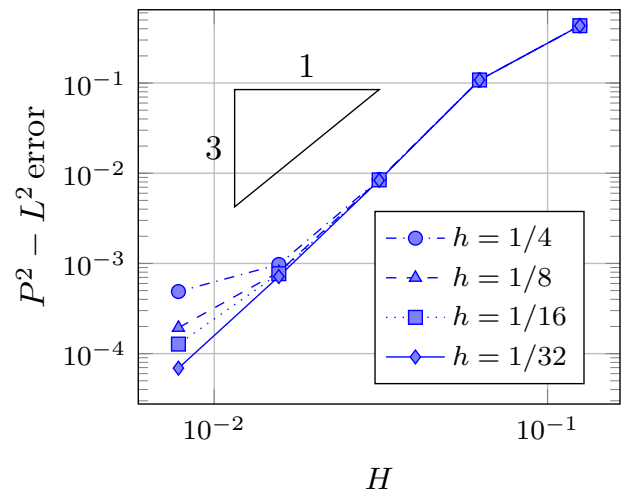

(b)

Figure 6: Locally periodic medium. Error $\left\|u^{0}(T)-u^{H}(T)\right\|$ vs. the macro mesh size $H$ for different fixed micro mesh sizes $h$ with $P^{2}$ macro and $P^{1}$ micro FE spaces: (a) $H^{1}(\Omega)$ and (b) $L^{2}(\Omega)$ norm.

\section{Horizontally layered material}

We now consider a horizontally layered HTI-VTI material ([16]), where the tensor $a^{\varepsilon}$ is given by

$$
a^{\varepsilon}(x)=a_{I} \chi^{\varepsilon}(x)+a_{I I}\left(1-\chi^{\varepsilon}(x)\right), \quad x \in \Omega,
$$

and $\chi^{\varepsilon}(x)$ corresponds to the indicator function of the $\varepsilon$-width horizontal layers shown in Figures $7(\mathrm{a})$ and $7(\mathrm{~b})$; hence, $\chi^{\varepsilon}(x)$ is constant in the (horizontal) $x_{1}$-direction and alternates between 0 and 1 in the $x_{2}$ direction.

We let $\Omega=[-2,0]^{2}$, where $x_{2}=0$ corresponds to the earth's surface, and set the tensors $a_{V}$ and $a_{H}$ to

$$
a_{I}=\left(\begin{array}{ccc}
46 & 18 & 0 \\
18 & 30 & 0 \\
0 & 0 & 7
\end{array}\right), \quad a_{I I}=\left(\begin{array}{ccc}
30 & 18 & 0 \\
18 & 46 & 0 \\
0 & 0 & 7
\end{array}\right) .
$$

Note that the tensor $a_{I}$ has horizontal anisotropy, whereas the tensor $a_{I I}$ has vertical anisotropy. For $\varepsilon=1 / 10$, the component $a_{1111}^{\varepsilon}$ and $a_{2222}^{\varepsilon}$ are represented in Figures $7(\mathrm{a})$ and $7(\mathrm{~b})$ respectively. We compute the homogenized tensor on a cell problem of size $\delta=2 \varepsilon$ with a mesh size $h=\frac{1}{1024}$, in accordance with the numerical results of [24].

For horizontally layered anisotropy elastic media, an effective homogeneous tensor can be derived using an averaging method proposed by Schoenberg and Muir [37]. Detailed equations for the computations of the homogenized tensor can be found in [16], which yield

$$
a^{0}=\left(\begin{array}{ccc}
38 & 18 & 0 \\
18 & 36.30 & 0 \\
0 & 0 & 7
\end{array}\right)
$$

The numerical homogenized tensor $a_{K}^{0}$ can be computed during the assembly process of the FE-HMM using equation (15). As the medium is periodic, the value of the numerical homogenized tensor $a_{K}^{0}$, computed in the sampling domain $K_{\delta_{j}}$, is the same at each quadrature node $x_{j, K}$. 

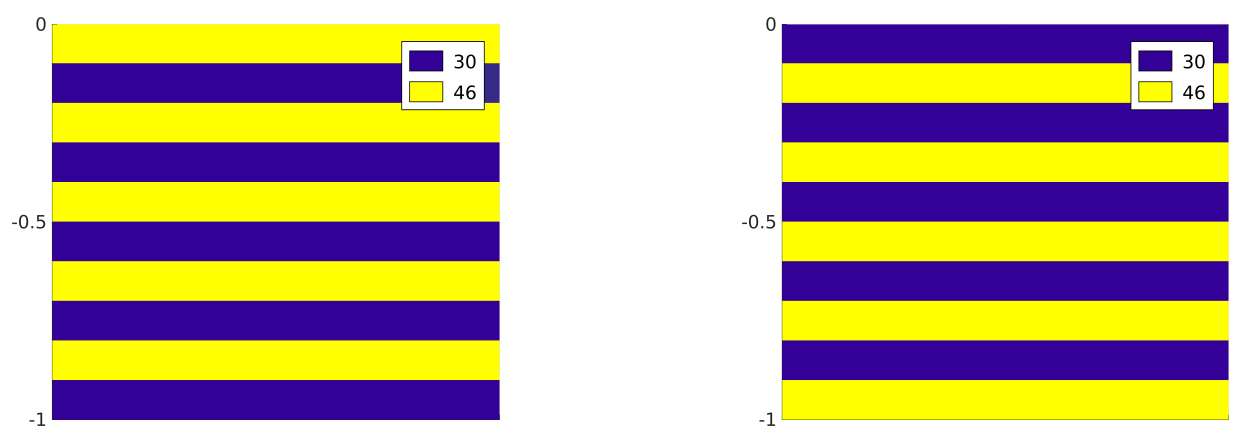

Figure 7: Horizontally layered material. Layered material components (a) $a_{1111}^{\varepsilon}$ and (b) $a_{2222}^{\varepsilon}$ for $\varepsilon=1 / 10$.

Using $P^{1}$ macro and micro FE, we obtain that $a_{K}^{0}$ is

$$
a_{K}^{0}=\left(\begin{array}{ccc}
38 & 18 & 0 \\
18 & 36.3158 & 0 \\
0 & 0 & 7
\end{array}\right),
$$

and the error $e=\left|a_{K}^{0}-a^{0}\right|$ in percent is

$$
e=\left(\begin{array}{ccc}
0 & 0 & 0 \\
0 & 0.04 \% & 0 \\
0 & 0 & 0
\end{array}\right)
$$

Using quadratic micro FE gives

$$
a_{K}^{0}=\left(\begin{array}{ccc}
37.99 & 17.99 & 0 \\
17.99 & 36.328 & 0 \\
0 & 0 & 7
\end{array}\right)
$$

and an error $e=\left|a_{K}^{0}-a^{0}\right|$ in percent of

$$
e=\left(\begin{array}{ccc}
0.026 \% & 0.055 \% & 0 \\
0.055 \% & 0.077 \% & 0 \\
0 & 0 & 0
\end{array}\right)
$$

In Figure 8(a), we plot the amplitude (in the Euclidean norm) of the reference solution $u^{\varepsilon}$ at time $t=0.1$ for $\varepsilon=1 / 50$ and in Figure 8(b), we plot the amplitude of the numerical homogenized solution. The global behavior of the two solutions is similar.

\section{Arbitrarily heterogeneous media}

In our final numerical experiment, we consider a random layered media generated by the von-Karman correlation function [26, 24, 33]

$$
\left.v_{\kappa}(x)=\frac{1}{2^{\kappa-1} \Gamma(|\kappa|)}\left(\left|\frac{x}{c}\right|\right)^{\kappa} K_{\kappa}\left(\left|\frac{x}{c}\right|\right)\right)
$$




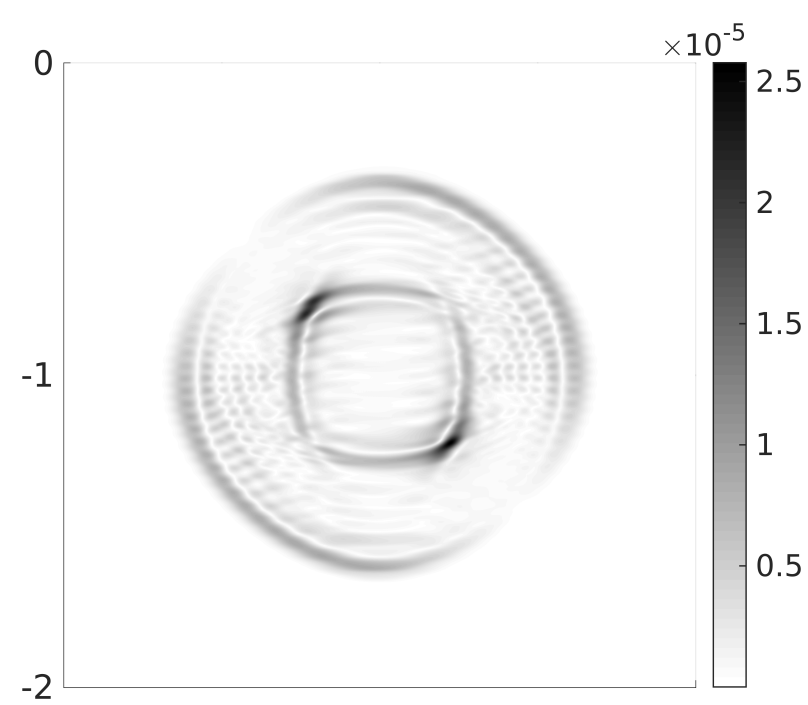

(a)

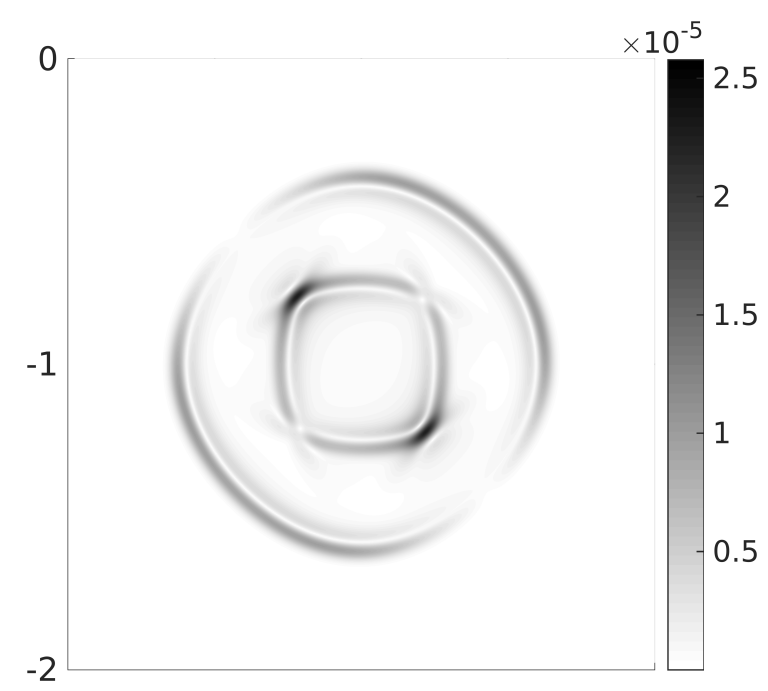

(b)

Figure 8: Horizontally layered material. Snapshots at time $t=0.1$ of (a) the amplitude of the reference solution $u^{\varepsilon}$ and (b) the amplitude of the homogenized solution for $\varepsilon=1 / 50$.

where $\kappa$ is the Hurst number, $K_{\kappa}$ is a modified Bessel function of order $\kappa$, and $c=\left(c_{H}, c_{V}\right)$ is the correlation distance of the heterogeneities in the medium, where $c_{H}\left(c_{V}\right)$ stands for the horizontal (vertical) size of the heterogeneities.

The medium is obtained by filtering white noise by a spectral filter, which is the square root of the power spectrum density function (the Fourier transform) of the von-Karman correlation function. We start by computing the von-Karman function on a fine grid over $\Omega$, compute its Fourier transform and take the square root; this is the spectral filter. Next, we take a uniform distribution between 0 and 1, compute its Fourier transform and multiply it by the filter. The random field, denoted by $\xi^{\varepsilon}$, is then obtained by transforming back to the spatial domain $\Omega$. We chose $\kappa<0$, as in such cases the von Karman correlation function describes small heterogeneities that are not resolved by the effective solution [30]. The tensor $a^{\varepsilon}$ is given by adding the random von-Karman field to a layered tensor with layer size of $\varepsilon$, i.e.,

$$
a^{\varepsilon}(x)=a_{L}^{\varepsilon}(x)+\xi^{\varepsilon} I_{3 \times 3} .
$$

The layered tensor, denoted by $a_{L}^{\varepsilon}$, is given by

$$
a_{L}^{\varepsilon}=\left(\begin{array}{ccc}
a_{1111} & a_{1122} & 0 \\
a_{1122} & a_{2222} & 0 \\
0 & 0 & a_{1212}
\end{array}\right),
$$

where each entry is layered. The tensor $a^{\varepsilon}$ is the three-by-three matrix

$$
a^{\varepsilon}(x)=\left(\begin{array}{ccc}
a_{1111}+\xi^{\varepsilon} & a_{1122}+\xi^{\varepsilon} & \xi^{\varepsilon} \\
a_{2211}+\xi^{\varepsilon} & a_{2222}+\xi^{\varepsilon} & \xi^{\varepsilon} \\
\xi^{\varepsilon} & \xi^{\varepsilon} & a_{1212}+\xi^{\varepsilon}
\end{array}\right) .
$$




\begin{tabular}{|l||c|c|c|c|c|c|}
\hline$\delta=8 \varepsilon$ & 13.5734 & 4.1497 & 0.6353 & 11.0198 & 1.4192 & 5.2596 \\
\hline$\delta=16 \varepsilon$ & 13.5824 & 4.1541 & 0.6377 & 11.0261 & 1.4265 & 5.2665 \\
\hline$\delta=24 \varepsilon$ & 13.5653 & 4.137 & 0.6277 & 11.0144 & 1.4028 & 5.2475 \\
\hline$\delta=32 \varepsilon$ & 13.5822 & 4.1538 & 0.6328 & 11.0600 & 1.4137 & 5.2723 \\
\hline$\delta=40 \varepsilon$ & 13.5759 & 4.1512 & 0.6354 & 11.0331 & 1.4216 & 5.2662 \\
\hline$\delta=48 \varepsilon$ & 13.5751 & 4.1534 & 0.6353 & 11.0453 & 1.4218 & 5.2712 \\
\hline
\end{tabular}

Table 1: Layered von-Karman random medium. Components of the numerical homogenized tensor computed on sampling domains with increasing size $\delta$.

Let $K_{\kappa}$ be the first order modified Bessel function and set $\kappa=-0.2, c=(0.2,0.01)$, and $\varepsilon=1 / 16$. The different components of the tensor $a^{\varepsilon}$ are shown in Figures 9 (a) to $9(\mathrm{f})$.

As the tensor has a period of four layers, we take sampling domains of size $\delta=4 n \varepsilon$, $n \in \mathbb{N} *$ with a mesh size $h$ small enough to capture the heterogeneities of the media, i.e., set by the correlation distance $c$. The Schoenberg-Muir averaging method fails for layered media with arbitrary heterogeneities, and similarly we cannot use the exact formula for the homogeneous tensor in a layered media [29, 19]. Thus, no explicit equations are available for the homogenized tensor and its values are sensitive to the size of the sampling domains, $\delta$. However, from the theory of homogenization in random media, the homogenized tensor at a point $x \in \Omega$ reaches a stable value as $\delta$ increases. We take a micro number of degrees of freedom $N_{\text {micro }}=1025$ and increase $\delta$. We set $\varepsilon=1 / 50, c=(\varepsilon / 2, \varepsilon / 4)$ and take $n=$ $2,3, \ldots, 12$, leading to sampling domain of sizes $8 \varepsilon \leq \delta<1$. We take a quadrature point $x_{j, K}$, at the center of $\Omega$, and compute the numerical homogenized tensor $a_{K}^{0}\left(x_{j, K}\right)$. As $\delta$ increases while keeping a mesh size to fully resolve the fine scales, the numerical tensor tends to stabilize around

$$
a_{K}^{0}\left(x_{j, K}\right)=\left(\begin{array}{ccc}
13.575 & 4.153 & 0.635 \\
4.153 & 11.045 & 1.421 \\
0.635 & 1.421 & 5.271
\end{array}\right) .
$$

Table 5 gives the values of the numerical homogenized tensor $a_{K}^{0}\left(x_{j, K}\right)$ for different values of $\delta$.

Now, we consider homogeneous Neumann boundary conditions and an initial condition given by a Gaussian pulse located at the center of $\Omega$. For both the reference and the numerical solutions, we consider a CFL condition

$$
\Delta t \leq \frac{h_{f}}{30}
$$

Let $u^{H}$ be the numerical homogenized solution computed with FE-HMM using $P^{2}$ macro FE and $P^{1}$ micro FE with $\delta=8 \varepsilon$ with a number of micro degrees of freedom $N_{\text {micro }}=128$, 


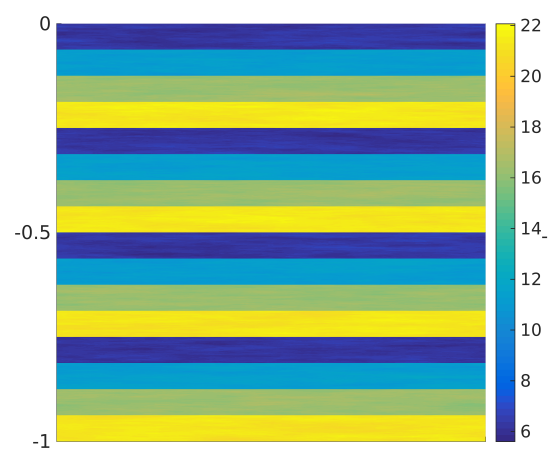

(a)

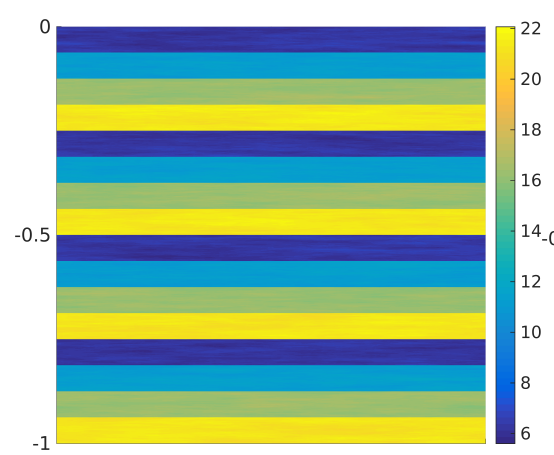

(d)

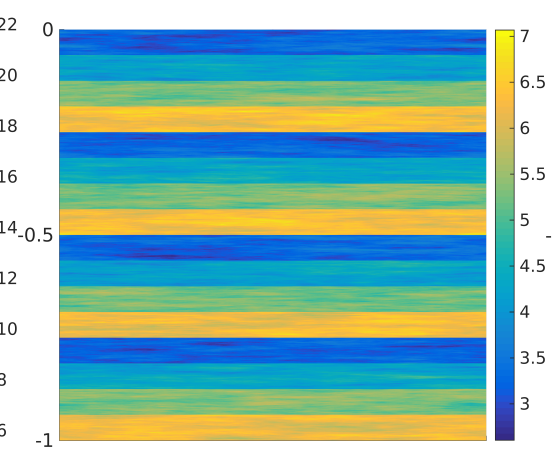

(b)

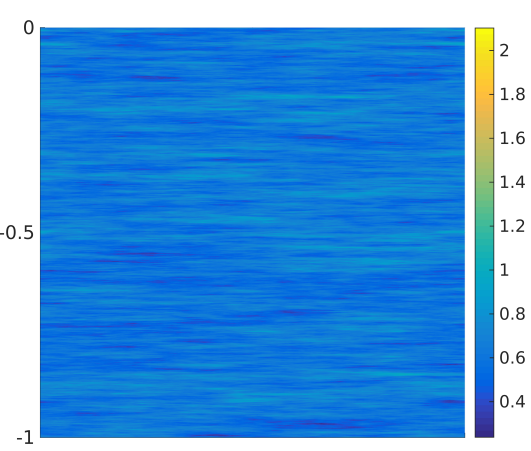

(c)

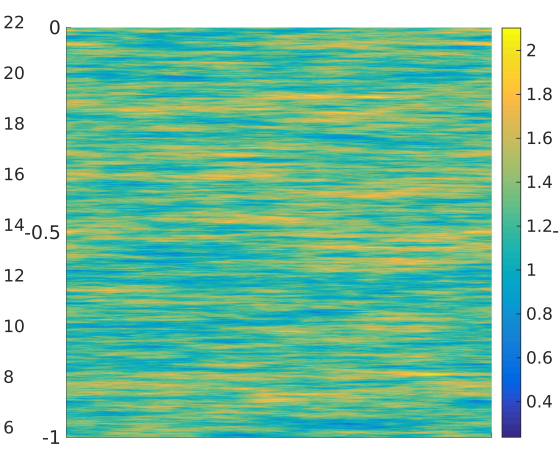

(e) Random field $\xi^{\varepsilon}$

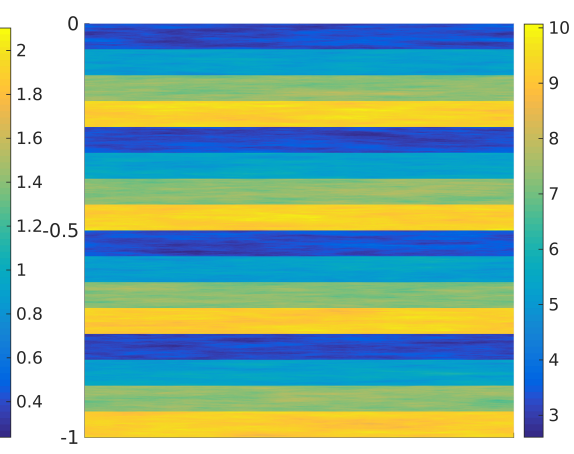

(f)

Figure 9: Layered von-Karman random media for $\varepsilon=1 / 16$, (a) $a_{1111}^{\varepsilon}$, (b) $a_{1122}^{\varepsilon}$, (c) $a_{1112}^{\varepsilon}$, (d) $a_{2222}^{\varepsilon}$, (e) $a_{2212}^{\varepsilon}$, and (f) $a_{1212}^{\varepsilon}$. 
where the micro problems are solved around each macro quadrature points. Consider as well, a numerical solution $u^{0, H}$ computed with the tensor (25) obtained by taking a sampling domain of size $\delta=48 \varepsilon$ with a number of micro degrees of freedom $N_{\text {micro }}=1024$. In Figure 10 , we plot snapshots at times $t=0.025,0.05,0.075$, and 0.1 of the numerical homogenized solution $u^{H}$ in the first column, the numerical solution $u^{0, H}$ in the second column, and the reference solution $u^{\varepsilon}$ in the third column. We observe that the three solutions give similar behavior, albeit with oscillations for the reference solution. At last, in Figure 11, we plot the reference solution $u^{\varepsilon}$ for $\varepsilon=1 / 100$ at time $t=0.05$ and $t=0.1$. When $\varepsilon$ diminishes, we finde that the heterogeneous and homogeneous displacements essentially coincide.

In Figure 12, we compare two numerical FE-HMM solutions using a numerically homogenized tensor computed either on a single sampling domain (left column) or in each sampling domains of the macroscopic mesh (right column), each with $\delta=8 \varepsilon$. Both numerical solutions, computed using $P^{2}$-macro and $P^{1}$-micro $\mathrm{FE}$, yield similar displacement fields. In Figure 13, we also display horizontal cross-section at $x_{2}=-0.5$ in the $x_{1}$-direction and at $x_{1}=0$ in the $x_{2}$-direction. In Figures $13(\mathrm{a})$ and 13(b), we compare the numerical homogenized solutions computed with a numerical tensor $a_{K}^{0}$ obtained from a single sampling domain $K_{\delta_{j}}$ either with $\delta=48 \varepsilon$ (in blue) or with $\delta=8 \varepsilon$ (in black). Further, in Figures $13(\mathrm{c})$ and $13(\mathrm{~d})$, we compare the numerical homogenized solutions computed either with $a_{K}^{0}$ obtaind from a single sampling domain $K_{\delta_{j}}$ (in black) or from individual sampling domains $K_{\delta_{j}}$, both with $\delta=8 \varepsilon$. All numerical solutions essentially coincide.

\section{Conclusion}

We have presented a multiscale FE method for time-dependent wave propagation in heterogeneous elastic media. It is based on a macro FE discretization of an effective wave equation, whose coefficients are computed on local sampling domains through micro computations. Although the finite element heterogeneous mutiscale method (FE-HMM) assumes that the heterogeneities occur at a micro scale $\varepsilon>0$ smaller than the dominating wave length, it neither requires the small-scale dependence to be periodic nor does it make any simplifying assumptions about the macro scale behavior of the elastic medium.

Since the size of the sampling domains scales with the smallest scale, $\varepsilon$, in the medium, our method accurately captures the overall macroscopic behavior of the time-dependent wave field up to any given but fixed time at a computational cost independent $\varepsilon$. We have proved fully discrete a priori error estimates in the $H^{1}$ and $L^{2}$ norm, which yield optimal convergence rates when the macro and micro mesh parameters are refined simultaneously. Our numerical experiments in locally periodic or stochastic media corroborate the expected convergence rates but also demonstrate the accuracy in fully heterogeneous situations.

Since the FE-HMM approach is based on standard finite elements at the macro and micro levels, it immediately applies to higher order finite or spectral elements, and to higher dimensional problems. For added flexibility in the mesh design, discontinuous Galerkin methods can also be used instead [3, 28]. Since the FE-HMM approach preserves the structure of the mass-matrix resulting from the macro FE discretization, it enables explicit time integration, just like standard FEM. Hence in the presence of complex geometry and local mesh 

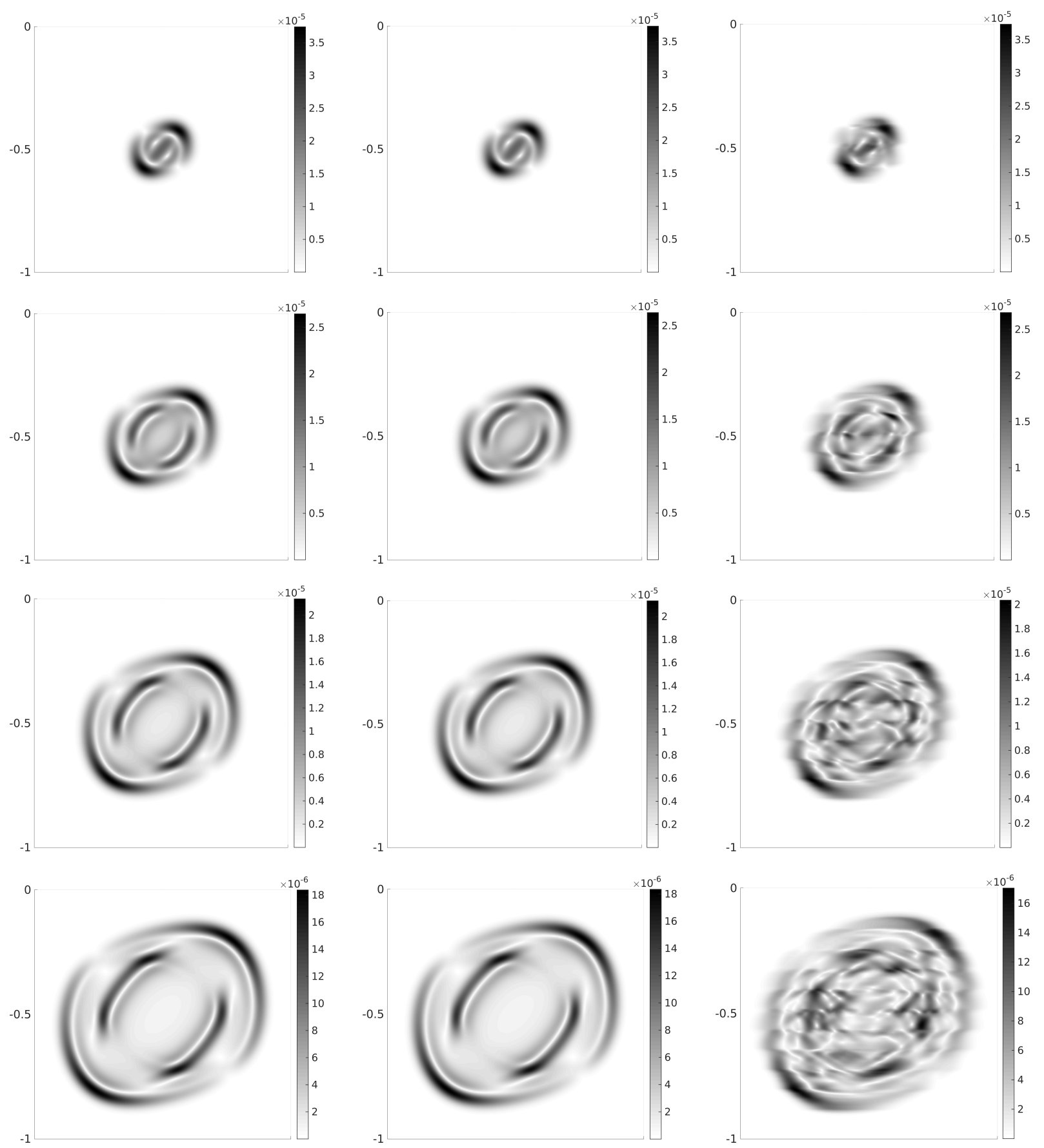

Figure 10: Layered von-Karman random medium. Snapshots at time $t=0.025,0.05,0.075$, and 0.1 of the amplitudes of the numerical homogenized solution ( $1^{\text {st }}$ column), the homogeneous solution with the tensor (25) ( $2^{\text {nd }}$ column), and the reference heterogeneous solution $u^{\varepsilon}\left(3^{r d}\right.$ column). 

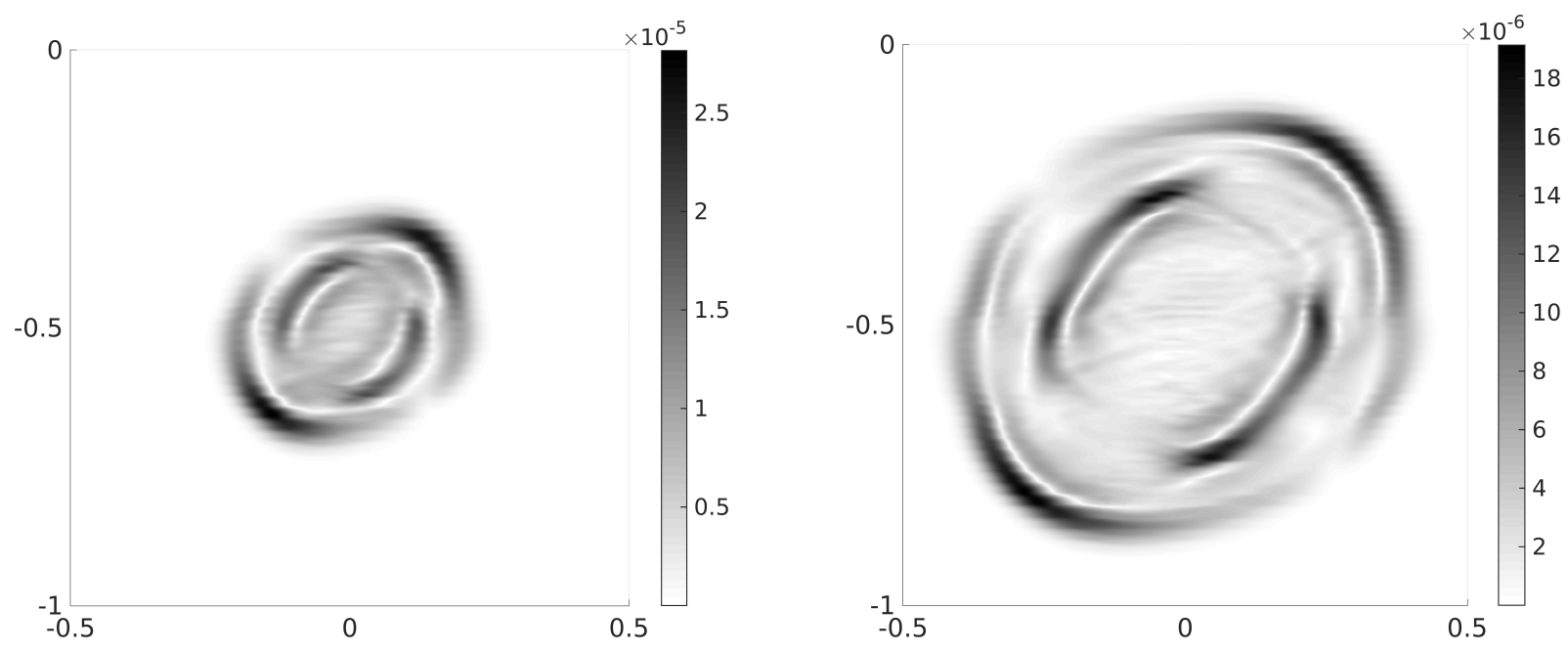

Figure 11: Layered von-Karman random medium. Snapshots at time $t=0.05$ and 0.1 of the amplitude of the reference solution $u^{\varepsilon}$ for $\varepsilon=1 / 100$.

refinement at the macroscopic level, it can also be combined with explicit local time-stepping strategies [20, 27].

The FE-HMM approach can also be extended to capture long time behavior by using appropriate modified effective equations, see [10] and the references therein.

Acknowledgements This work of A. Abdulle and O. Jecker was partially supported by the Fonds National Suisse, project No. 200021-150019.

[1] AbDulle, A. Analysis of a heterogeneous multiscale FEM for problems in elasticity. Math. Models Methods Appl. Sci. 16, 4 (2006), 615-635.

[2] AbDulle, A. The finite element heterogeneous multiscale method: a computational strategy for multiscale PDEs. In Multiple scales problems in biomathematics, mechanics, physics and numerics, vol. 31 of GAKUTO Internat. Ser. Math. Sci. Appl. Gakkōtosho, Tokyo, 2009, pp. 133-181.

[3] Abdulle, A. Discontinuous Galerkin finite element heterogeneous multiscale method for elliptic problems with multiple scales. Math. Comp. 81, 278 (2012), 687-713.

[4] Abdulle, A., And E, W. Finite difference heterogeneous multi-scale method for homogenization problems. J. Comput. Phys. 191, 1 (2003), 18-39.

[5] Abdulle, A., E, W., Engquist, B., And Vanden-Eijnden, E. The heterogeneous multiscale method. Acta Numer. 21 (2012), 1-87.

[6] Abdulle, A., And Grote, M. J. Finite element heterogeneous multiscale method for the wave equation. Multiscale Model. Simul. 9, 2 (2011), 766-792. 

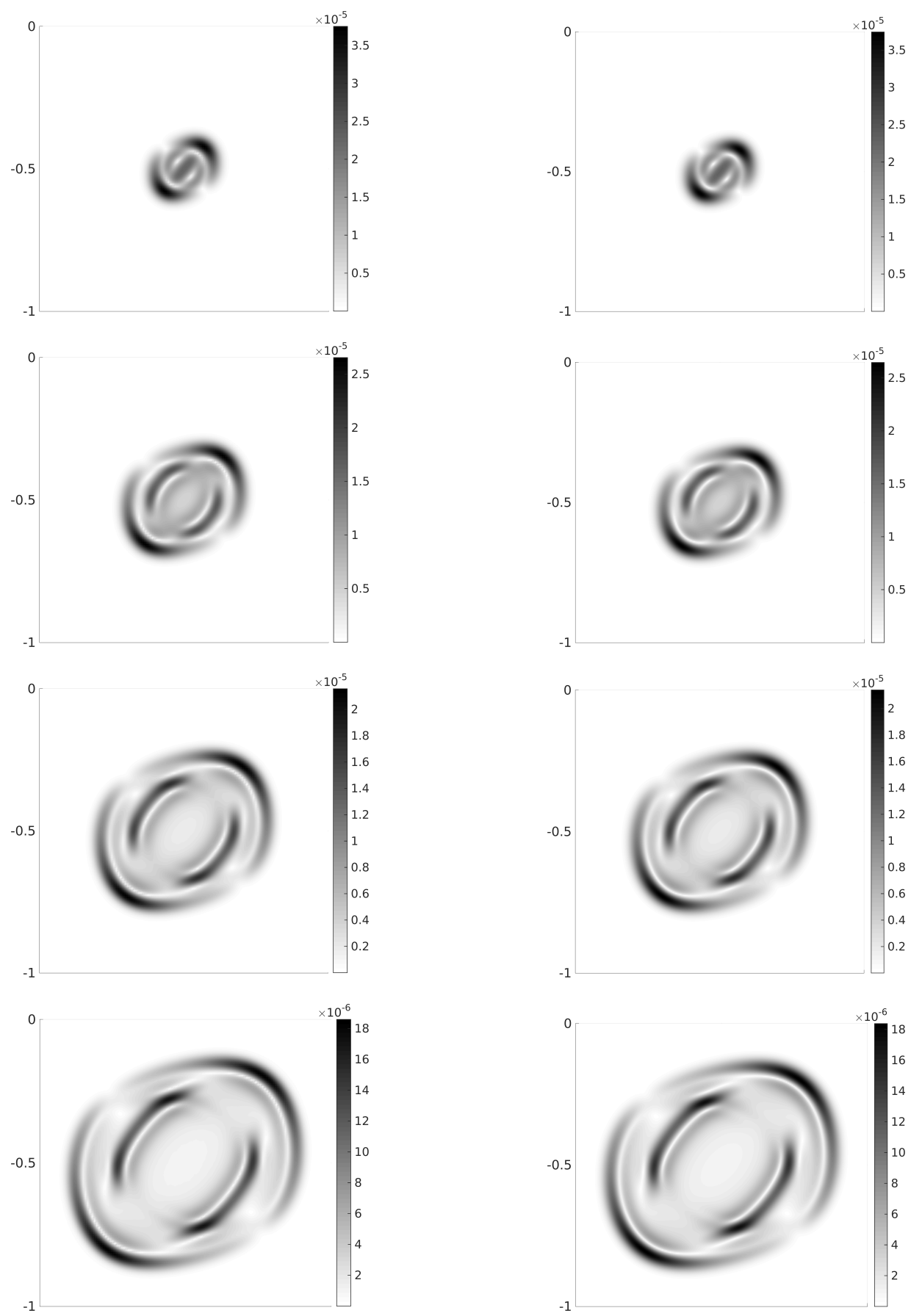

Figure 12: Layered von-Karman random medium. Snapshots at time $t=0.025,0.05,0.075$, and 0.1 of the numerical homogenized solution with $a_{K}^{0}$ computed in one sampling domain $K_{\delta_{j}}$ with $\delta=8 \varepsilon$, and whose value is used at each quadrature points ( $1^{\text {st }}$ column), snapshots of the homogeneous solution with $a_{K}^{0}$ computed in each sampling domain $K_{\delta_{j}}$ with $\delta=8 \varepsilon$ ( $2^{\text {nd }}$ column). 


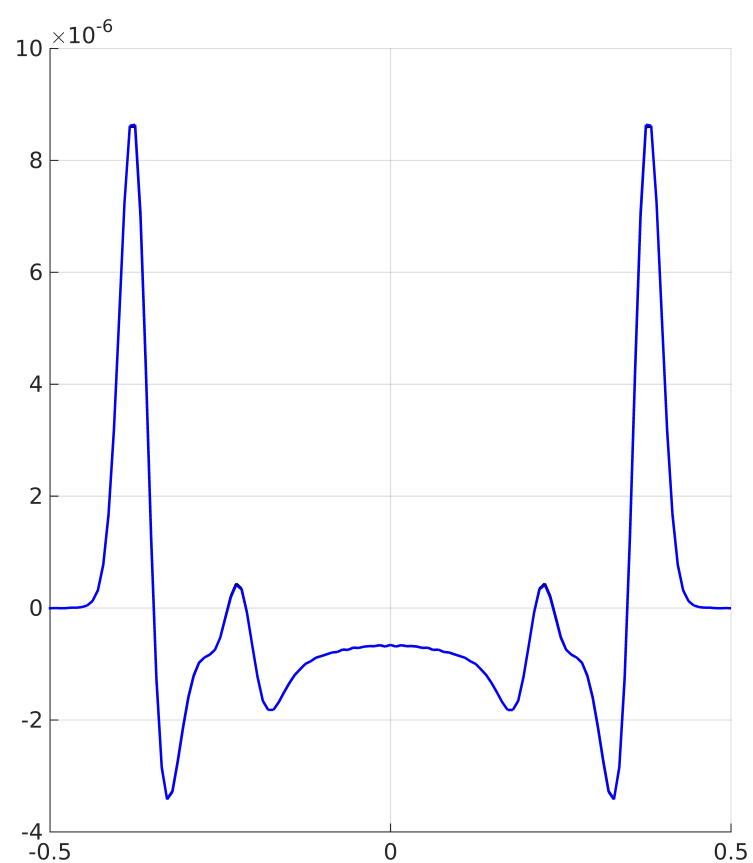

(a)

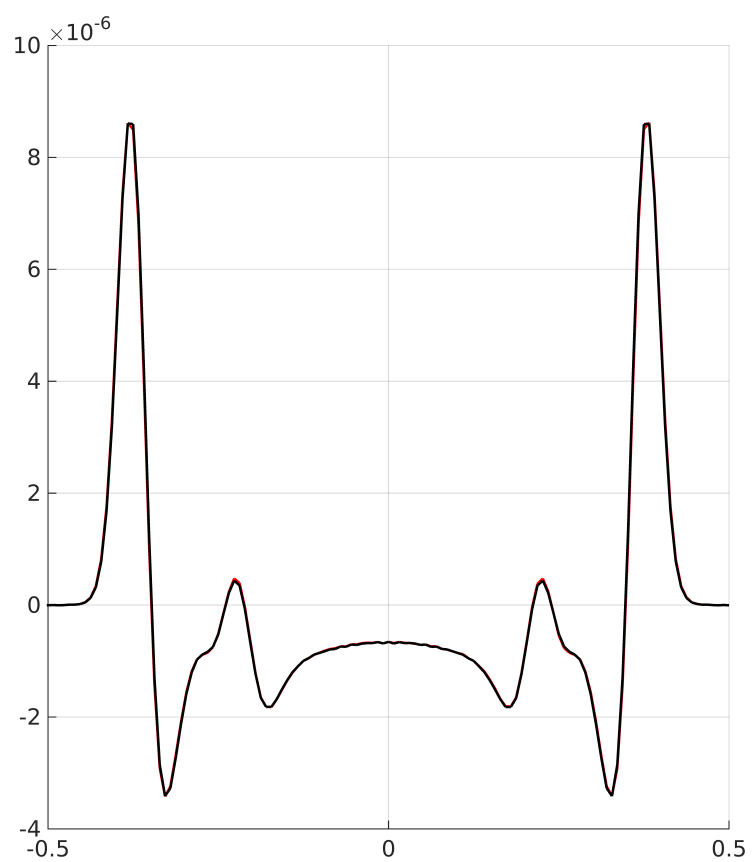

(c)

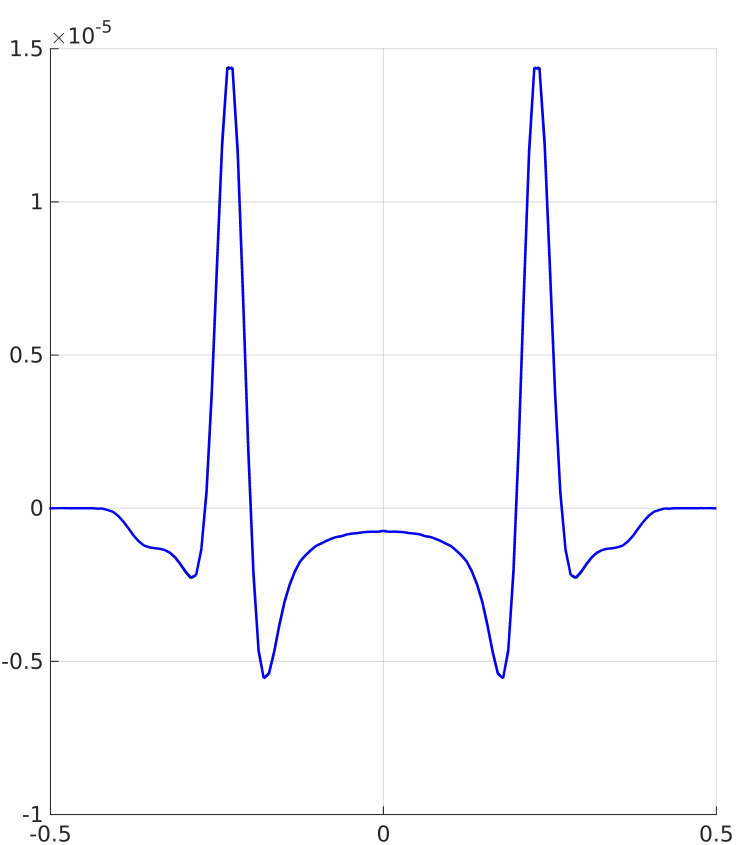

(b)

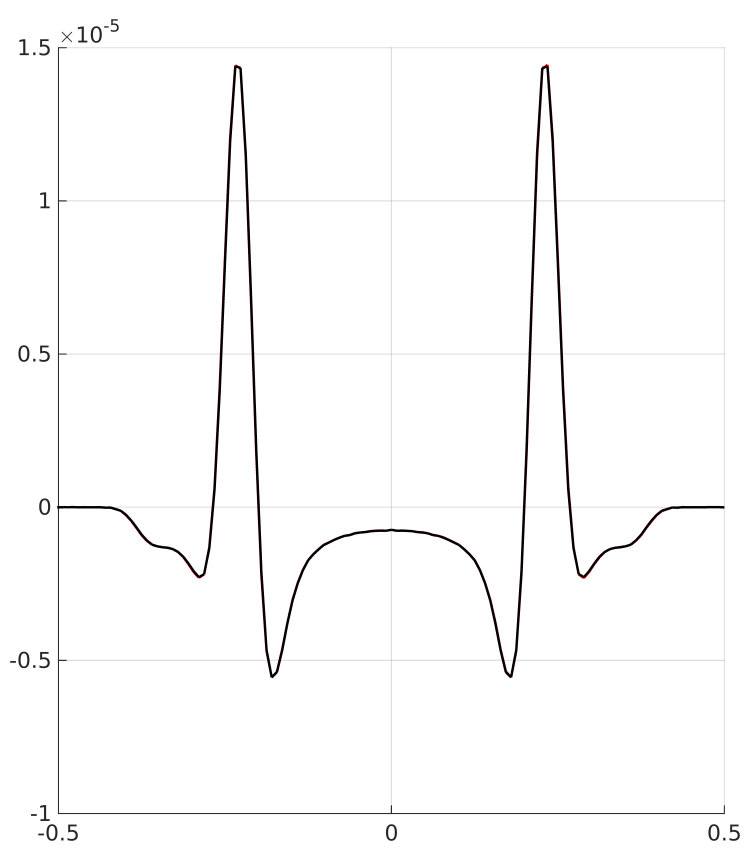

(d)

Figure 13: Layered von-Karman random medium. Horizontal cross-sections at time $t=0.1$ of the (a) $x_{1}$ direction at depth $x_{2}=-0.5$ and (b) at $x_{1}=0$ in the $x_{2}$-direction of the numerical homogenized solution with $a_{K}^{0}$ computed in one sampling domain $K_{\delta_{j}}$ with $\delta=48 \varepsilon$ (in blue) and with $\delta=8 \varepsilon$ (in black). Horizontal cross-section of the (c) $x_{1}$-direction at depth $x_{2}=-0.5$ and (d) $x_{2}$-direction at $x_{1}$-direction of the numerical homogenized solution with $a_{K}^{0}$ computed in one sampling domain $K_{\delta_{j}}$ with $\delta=8 \varepsilon$ (in black) and the numerical homogenized solution with $a_{K}^{0}$ computed in each sampling domain $K_{\delta_{j}}$ with $\delta=8 \varepsilon$ (in red). 
[7] Abdulle, A., Grote, M. J., And Stohrer, C. Finite element heterogeneous multiscale method for the wave equation: long-time effects. Multiscale Model. Simul. $\underline{12}, 3$ (2014), 1230-1257.

[8] Abdulle, A., And Henning, P. Chapter 20 - multiscale methods for wave problems in heterogeneous media. In Handbook of Numerical Methods for Hyperbolic Problems Applied and Modern Issues, R. Abgrall and C.-W. Shu, Eds., vol. 18 of Handbook of Numerical Analysis. Elsevier, 2017, pp. 545-576.

[9] Abdulle, A., And Jecker, O. Numerical experiments for multiscale problems in linear elasticity. In Numerical Mathematics and Advanced Applications ENUMATH 2015 (2016), Springer, pp. 123-131.

[10] Abdulle, A., And Pouchon, T. A priori error analysis of the finite element heterogeneous multiscale method for the wave equation over long time. SIAM J. Numer. Anal. 54, 3 (2016), 1507-1534.

[11] Allaire, G. Shape Optimization by the Homogenization Method. Applied Mathematical Sciences, 146. Springer-Verlag New York, 2002.

[12] Arjmand, D., And Runborg, O. Analysis of heterogeneous multiscale methods for long time wave propagation problems. Multiscale Model. Simul. 12, 3 (2014), 1135-1166.

[13] Bensoussan, A., Lions, J.-L., and Papanicolaou, G. Asymptotic analysis for periodic structures. North-Holland Publishing Co., Amsterdam, 1978.

[14] Capdeville, Y., Guillot, L., and Marigo, J.-J. 1-d non-periodic homogenization for the seismic wave equation. Geophys. J. Int. 181, 2 (2010), 897-910.

[15] Capdeville, Y., Zhao, M., and Cupillard, P. Fast fourier homogenization for elastic wave propagation in complex media. Wave Motion 54 (2015), 170-186.

[16] Carcione, J. M., Picotti, S., Cavallini, F., and Santos, J. E. Numerical test of the schoenberg-muir theory. Geophysics 77, 2 (2012), C27-C35.

[17] Ciarlet, P. G. The finite element method for elliptic problems, vol. 4 of Studies in Mathematics and its Applications. North-Holland, 1978.

[18] Ciarlet, P. G., And Raviart, P. A. The combined effect of curved boundaries and numerical integration in isoparametric finite element methods. In The mathematical foundations of the finite element method with applications to partial differential equations (1972), pp. 409-474.

[19] Cioranescu, D., And Donato, P. An introduction to homogenization, vol. 17 of Oxford Lecture Series in Mathematics and its Applications. Oxford University Press, New York, 1999. 
[20] Diaz, J., And Grote, M. J. Energy conserving explicit local time stepping for secondorder wave equations. SIAM Journal on Scientific Computing 31, 3 (2009), 1985-2014.

[21] E, W., And Engquist, B. The heterogeneous multiscale methods. Commun. Math. Sci. 1, 1 (2003), 87-132.

[22] Engquist, B., Holst, H., And Runborg, O. Multi-scale methods for wave propagation in heterogeneous media. Commun. Math. Sci. 9, 1 (2011), 33-56.

[23] Engquist, B., Holst, H., And Runborg, O. Multiscale methods for wave propagation in heterogeneous media over long time. In Numerical analysis of multiscale computations, vol. 82 of Lect. Notes Comput. Sci. Eng. Springer, Heidelberg, 2012, pp. 167-186.

[24] Gao, K., Chung, E. T., Gibson JR, R. L., Fu, S., and Efendiev, Y. A numerical homogenization method for heterogeneous, anisotropic elastic media based on multiscale theory. Geophysics 80, 4 (2015), D385-D401.

[25] Gao, K., Fu, S., Gibson, R. L., Chung, E. T., and Efendiev, Y. Generalized multiscale finite-element method (gmsfem) for elastic wave propagation in heterogeneous, anisotropic media. Journal of Computational Physics 295 (2015), 161-188.

[26] Goff, J. A., And Jordan, T. H. Stochastic modeling of seafloor morphology: Inversion of sea beam data for second-order statistics. Journal of Geophysical Research: Solid Earth 93, B11 (1988), 13589-13608.

[27] Grote, M. J., Mehlin, M., and Mitkova, T. Runge-Kutta-based explicit local time-stepping methods for wave propagation. SIAM journal on scientific computing 37, 2 (2015), A747-A775.

[28] Grote, M. J., Schneebeli, A., And Schötzau, D. Discontinuous Galerkin finite element method for the wave equation. SIAM Journal on Numerical Analysis 44, 6 (2006), 2408-2431.

[29] Jikov, V. V., Kozlov, S. M., and Oleinik, O. A. Homogenization of differential operators and integral functionals. Springer-Verlag, Berlin, Heidelberg, 1994.

[30] KLImeš, L. Correlation functions of random media. Pure and applied geophysics 159, 7-8 (2002), 1811-1831.

[31] Lions, J.-L., And Magenes, E. Problèmes aux limites non homogènes et applications, vol. 1 of Travaux et recherches mathématiques. Dunod, Paris, 1968.

[32] Murat, F., And Tartar, L. $H$-convergence. In Topics in the mathematical modelling of composite materials, vol. 31 of Progr. Nonlinear Differential Equations Appl. Birkhäuser Boston, Boston, MA, 1997, pp. 21-43. 
[33] Obermann, A., Planès, T., Larose, E., Sens-Schönfelder, C., And CAmpillo, M. Depth sensitivity of seismic coda waves to velocity perturbations in an elastic heterogeneous medium. Geophysical Journal International 194, 1 (2013), $372-382$.

[34] Oleinik, O. A., Shamaev, A., And Yosifian, G. Mathematical Problems in Elasticity and homogenization. North-Holland, Amsterdam, 1992.

[35] Owhadi, H., And Zhang, L. Numerical homogenization of the acoustic wave equations with a continuum of scales. Comput. Methods Appl. Mech. Engrg. 198, 3 (2008), 397-406.

[36] Rietmann, M., Grote, M., Peter, D., And Schenk, O. Newmark local time stepping on high-performance computing architectures. Journal of Computational Physics $\underline{334}$ (2017), 308-326.

[37] Schoenberg, M., And Muir, F. A calculus for finely layered anisotropic media. Geophysics 54, 5 (1989), 581-589.

[38] Vdovina, T., Minkoff, S. E., And Griffith, S. M. L. A two-scale solution algorithm for the elastic wave equation. SIAM J. Sci. Comput. 31, 5 (2009), 3356-3386. 


\section{LATEST PREPRINTS}

No. Author: Title

2016-33 S. Iula

A note on the Moser-Trudinger inequality in Sobolev-Slobodeckij spaces in dimension one

2016-34 C. Bürli, H. Harbrecht, P. Odermatt, S. Sayasone, N. Chitnis

Mathematical analysis of the transmission dynamics of the liver fluke, Opisthorchis viverrini

2017-01 J. Dölz and T. Gerig, M. Lüthi, H. Harbrecht and T. Vetter Efficient computation of low-rank Gaussian process models for surface and image registration

2017-02 M. J. Grote, M. Mehlin, S. A. Sauter

Convergence analysis of energy conserving explicit local time-stepping methods for the wave equation

2017-03 Y. Bilu, F. Luca, D. Masser

Collinear CM-points

2017-04 P. Zaspel

Ensemble Kalman filters for reliability estimation in perfusion inference

2017-05 J. Dölz and H. Harbrecht

Hierarchical Matrix Approximation for the Uncertainty Quantification of

Potentials on Random Domains

2017-06 P. Zaspel

Analysis and parallelization strategies for Ruge-Stüben AMG on many-core processor

2017-07 H. Harbrecht and M. Schmidlin

Multilevel Methods for Uncertainty Quantification of Elliptic PDEs with Random Anisotropic Diffusion

2017-08 M. Griebel and H. Harbrecht

Singular value decomposition versus sparse grids: Refined complexity

Estimates

2017-09 J. Garcke and I. Kalmykov

Efficient Higher Order Time Discretization Schemes for Hamilton-JacobiBellman Equations Based on Diagonally Implicit Symplectic Runge-Kutta Methods

2017-10 M. J. Grote and U. Nahum

Adaptive Eigenspace Regularization For Inverse Scattering Problems

Preprints are available under https://math.unibas.ch/research/publications 


\section{LATEST PREPRINTS}

No. Author: Title

2017-11 J. Dölz, H. Harbrecht, S. Kurz, S. Schöps and F. Wolf

A Fast Isogeometric BEM for the Three Dimensional Laplace- and Helmholtz Problems

2017-12 P. Zaspel

Algorithmic patterns for $\mathrm{H}$-matrices on many-core processors

2017-13 R. Brügger, R. Croce and H. Harbrecht

Solving a free boundary problem with non-constant coefficients

2017-14 M. Dambrine, H. Harbrecht and B. Puig

Incorporating knowledge on the measurement noise in electrical impedance tomography

2017-15 C. Bürli, H. Harbrecht, P. Odermatt, S. Sayasone and N. Chitnis Analysis of Interventions against the Liver Fluke, Opisthorchis viverrini

2017-16 D. W. Masser

Abcological anecdotes

2017-17 P. Corvaja, D. W. Masser and U. Zannier

Torsion hypersurfaces on abelian schemes and Betti coordinates

2017-18 F. Caubet, M. Dambrine and H. Harbrecht

A Newton method for the data completion problem and application to obstacle detection in Electrical Impedance Tomography

2018-01 H. Harbrecht and P. Zaspel

On the algebraic construction of sparse multilevel approximations of elliptic tensor product problems

2018-02 F. Ghiraldin and X. Lamy

Optimal Besov differentiability for entropy solutions of the eikonal equation

2018-03 H. Harbrecht and M. Schmidlin

Multilevel quadrature for elliptic problems on random domains by the coupling of FEM and BEM

2018-04 M. Bugeanu and H. Harbrecht

Parametric representation of molecular surfaces

2018-05 A. Abdulle, M. J. Grote and O. Jecker

Finite element heterogeneous multiscale method for Elastic Waves in

Heterogeneous Media

Preprints are available under https://math.unibas.ch/research/publications 